\title{
Multiresolution Time-Domain (MRTD) Adaptive Schemes Using Arbitrary Resolutions of Wavelets
}

\author{
Emmanouil M. Tentzeris, Member, IEEE, Andreas Cangellaris, Fellow, IEEE, Linda P. B. Katehi, Fellow, IEEE, \\ and James Harvey, Senior Member, IEEE
}

\begin{abstract}
A space- and time-adaptive two-dimensional multiresolution time-domain (MRTD) algorithm based on arbitrary resolutions of Battle-Lemarie wavelets is proposed. Analytic expressions for the finite-summation coefficients are derived and details concerning the modeling of hard boundaries, excitation, and field reconstruction are extensively discussed. Through the use of a combination of absolute and relative thresholding, a dynamically changing grid is developed with minimal computational requirements in comparison to the finite-difference time-domain technique. After the validation process, MRTD is used for the first time for the numerical optimization of complex RF structures such as evanescent-mode filters.
\end{abstract}

Index Terms-Adaptive gridding, FDTD, MRTD, time-domain techniques, wavelets.

\section{INTRODUCTION to TIME-DOMAIN TECHNIQUES}

$\mathbf{S}$ IGNIFICANT attention is being devoted today to the analysis and design of various types of printed components for microwave applications. Despite the wealth of available codes, many problems in electromagnetics, and specifically in circuit and antenna problems [e.g., monolithic microwave integrated circuit (MMIC) packaging, multichip modules (MCMs)], have been left untreated due to the complexity of the geometries and the inability of the existing techniques to deal with the requirements for large size and high resolution due to the fine, but electrically important geometrical details. For these cases, the straightforward use of existing discretization methods (such as finite difference time domain (FDTD) [1], [2]) suffers from serious limitations due to the required substantial computer resources and unrealistically long computation times. As a result, during the past 30 years, the available techniques are almost incapable of dealing with the needs of technology leading to a quest for fundamentally different modeling approaches. Recently, the use of scaling and wavelet functions as a com-

Manuscript received April 5, 2001. This work was supported by the National Science Foundation under the CAREER Award 9984761, by the State of Georgia under the Yamacraw Research Initiative, and by the Georgia Institute of Technology Packaging Research Center.

E. M. Tentzeris is with the School of Electrical and Computer Engineering, Georgia Institute of Technology, Atlanta, GA 30332 USA (e-mail: etentze@ece.gatech.edu).

A. Cangellaris is with the Department of Electrical and Computer Engineering, University of Illinois at Urbana-Champaign, Urbana, IL 61801 USA.

L. P. B. Katehi was with the Electrical Engineering and Computer Science Department, University of Michigan at Ann Arbor, Ann Arbor, MI 48109 USA She is now with the Dean of Schools of Engineering, Purdue University, West Lafayette, IN 47907 USA.

J. Harvey is with the Army Research Office, Research Triangle Park, NC 27709-2211 USA.

Publisher Item Identifier S 0018-9480(02)01161-4. plete set of field basis functions resulted in the multiresolution time-domain (MRTD) [3] schemes that are generalizations to Yee's FDTD and can extend the capabilities of the conventional FDTD by improving computational efficiency and substantially reducing computer resources by providing space and time adaptive gridding. Though various basis have been used [4]-[9], the Battle-Lemarie family has demonstrated the better economy in memory and execution time requirements, when only scaling functions have been used (cell size close to the Nyquist limit). The purpose of this paper is to extend the performance analysis to MRTD schemes based on Battle-Lemarie scaling and wavelets, exploit the adaptive character of such a scheme, and lay the foundation for the use of other expansion basis.

\section{FundAMENTALS OF MULTIRESOLUTION ANALYSIS}

One of the most important characteristics of expansion to scaling and wavelet functions is the time-frequency localization. The standard approach in ideal low-pass ("scaling") and bandpass ("wavelet") filtering for separating an analog signal into different frequency bands emphasizes the importance of time localization. The multiresolution analysis (MRA), introduced by Mallat [10] and Meyer [11], provides a very powerful tool for the construction of wavelets and implementation of the wavelet decomposition/reconstruction algorithms. The sampling theorem can be used to formulate analog signal representations in terms of superpositions of certain uniform shifts of a single function called a scaling function. Stability of this signal representation is achieved by imposing the Riesz condition on the scaling function. Another important condition of an MRA is the nested sequence of subspaces as a result of using scales by integer powers of two.

An MRA [12] consists of a sequence of successive approximation spaces $V_{j}$. More precisely, the closed subspaces $V_{j}$ satisfy

$$
\cdots V_{2} \subset V_{1} \subset V_{0} \subset V_{-1} \subset V_{-2} \subset \cdots
$$

with

$$
\begin{aligned}
& \overline{\bigcup_{j \in Z} V_{j}}=L^{2}(R) \quad \text { (density) } \\
& \bigcap_{j \in Z} V j=\{0\} \quad \text { (separation). }
\end{aligned}
$$

The basic idea of the MRA is that whenever a collection of closed subspaces satisfies the multiresolution conditions, there 
exists an orthonormal wavelet basis $\left\{\psi_{j, n}, n \in \mathbf{Z}\right\}$ of $L^{2}(\mathbf{R})$, $\psi_{j, n}(x)=2^{-j / 2} \psi\left(2^{-j} x-n\right)$, such that for all $f$ in $L^{2}(\mathbf{R})$

$$
P_{j-1} f=P_{j} f+\sum_{k \in Z}<f, \psi_{j, k}>\psi_{j, k}
$$

where $P_{j}$ is the orthogonal projection onto $V_{j}$. For every $j \in Z$, define $W_{j}$ to be the orthogonal complement of $V_{j}$ in $V_{j-1}$. We have

$$
V_{j-1}=V_{j} \oplus W_{j}
$$

and

$$
W_{j} \perp W_{j^{\prime}}, \quad j \neq j^{\prime} .
$$

It follows that for $j<J$

$$
V_{j}=V_{J} \oplus\left(\bigoplus_{k=0}^{J-j-1} W_{J-k}\right)
$$

where all these subspaces are orthogonal. Equation (7) is the foundation of multiresolution. Supposing that scaling functions of $J$ th-order approximation are used, the enhancement of wavelets of orders $J$ to $j+1$ create an approximation with much better accuracy (jth-order approximation). In other words, the scaling functions describe accurately the smooth features of a function and the wavelets the finer details for which a more accurate approximation has to be used. In this way, MRA operates as a "mathematical microscope." Wherever needed, a finite linear combination of wavelets can offer an arbitrarily small precision of the approximation.

It has to be noted that the $W_{j}$ spaces inherit the scaling property from the $V_{j}$

$$
f(x) \in W_{j} \leftrightarrow f\left(2^{j} x\right) \in W_{0} .
$$

The Battle-Lemarie wavelets [13], [14] based on the $m$ th order cardinal B-splines belong to $C^{k}$ with $k \leq m-1$, have very good (exponential) decay though the support equals the whole $R$, and have $m$ vanishing moments: $\int d x x^{l} \psi(x)=0$ for $l=$ $0,1, \ldots, m-1$ for $\psi^{(l)}$ bounded for $l \leq m$. The choices of the scaling function for the remainder of this paper are the cubic cardinal spline $(m=3)$. After orthonormalization, the spectral expressions of the cubic cardinal spline scaling and the wavelet functions (Figs. 1-4) are

$$
\begin{aligned}
& \hat{\phi}^{\perp}(\xi)=(2 \pi)^{-1 / 2}\left(\frac{\sin \left(\frac{\xi}{2}\right)}{\frac{\xi}{2}}\right)^{4} \\
& \cdot \frac{1}{\sqrt{1-\frac{4}{3} \sin ^{2}\left(\frac{\xi}{2}\right)+\frac{2}{5} \sin ^{4}\left(\frac{\xi}{2}\right)-\frac{4}{315} \sin ^{6}\left(\frac{\xi}{2}\right)}}
\end{aligned}
$$

and

$$
\hat{\psi}^{\perp}(\xi)=e^{i \xi / 2} \frac{\hat{\phi}^{\perp}(\xi+2 \pi)}{\hat{\phi}^{\perp}(\xi / 2+\pi)} \hat{\phi^{\perp}}(\xi / 2)=\hat{\psi}_{0}(\xi) .
$$

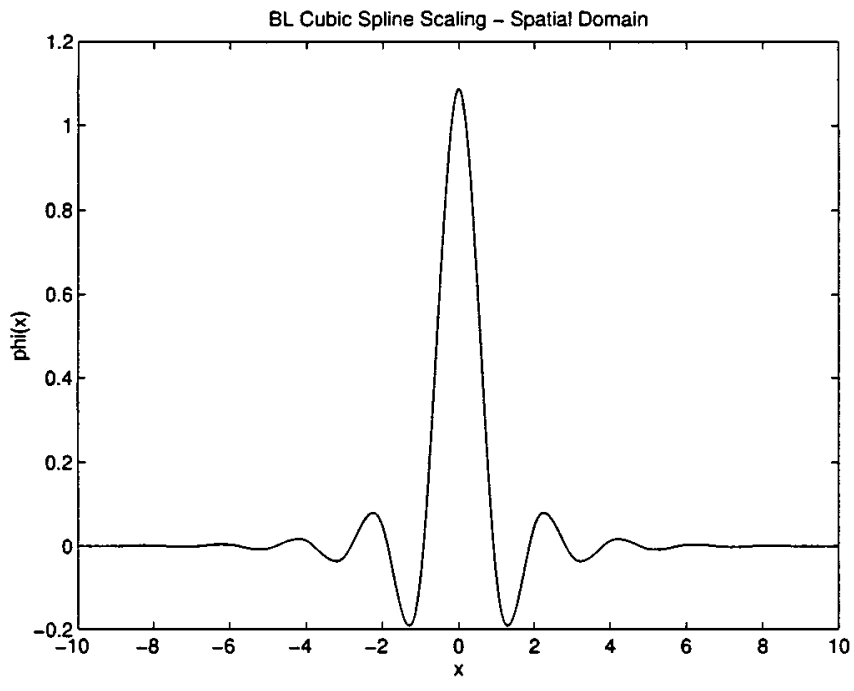

Fig. 1. Battle-Lemarie cubic spline scaling-spatial domain.

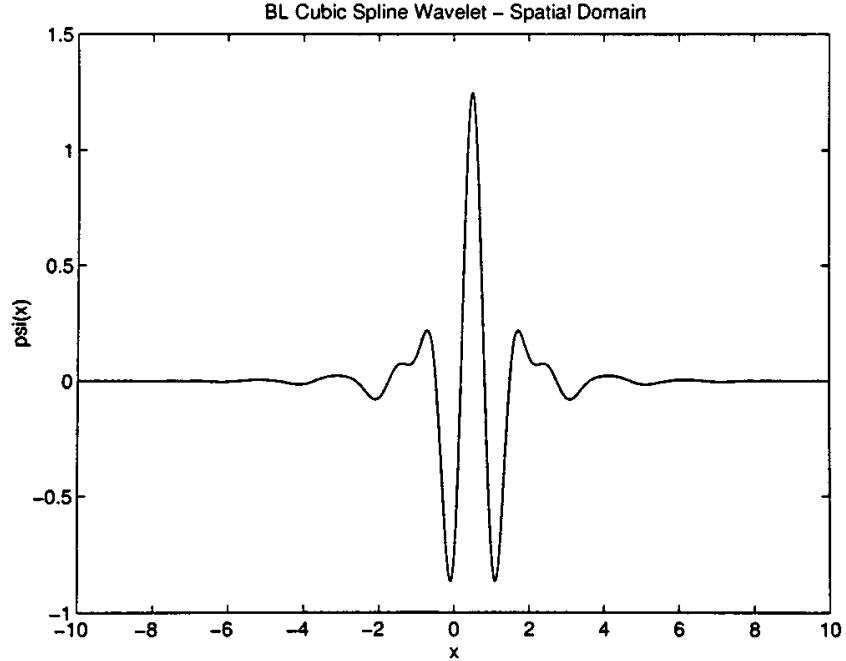

Fig. 2. Battle-Lemarie cubic spline zero-resolution wavelet—spatial domain.

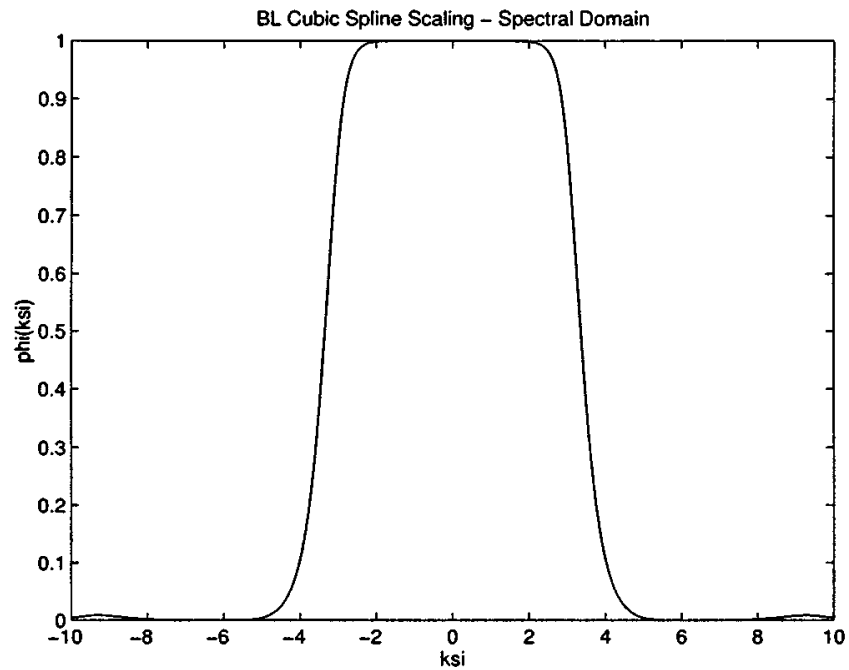

Fig. 3. Battle-Lemarie cubic spline scaling—spectral domain ("low-pass"). 


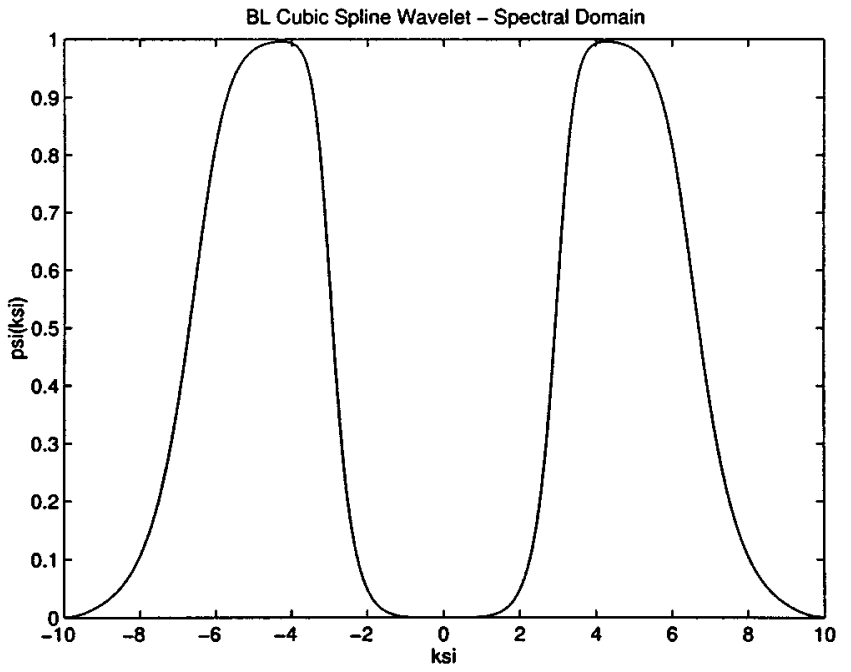

Fig. 4. Battle-Lemarie cubic spline zero-resolution wavelet-spectral domain ("bandpass").

\section{TWO-DIMENSIONAL MRTD SCHEME}

\section{A. Multiresolution Expansion}

For simplicity, the two-dimensional (2-D) MRTD scheme is analyzed for a homogeneous lossless medium with the permittivity $\epsilon$ and the permeability $\mu$. Assuming no variation along the $y$-direction, the Maxwell's equations for the 2-D $\mathrm{TM}^{z}$ mode [16] can be written as

$$
\begin{aligned}
\frac{\partial E_{x}}{\partial t} & =-\frac{1}{\epsilon} \frac{\partial H_{y}}{\partial z} \\
\frac{\partial H_{y}}{\partial t} & =\frac{1}{\mu}\left(\frac{\partial E_{z}}{\partial x}-\frac{\partial E_{x}}{\partial z}\right) \\
\frac{\partial E_{z}}{\partial t} & =\frac{1}{\epsilon} \frac{\partial H_{y}}{\partial x}
\end{aligned}
$$

To derive the 2-D MRTD scheme, the electric- and magneticfield components incorporated in these equations are expanded in a series of scaling and wavelet functions in both $x$ - and $z$-directions and in pulse functions in time [4], [17]

$$
\begin{aligned}
& E_{x}(x, z, t) \\
& =\sum_{k, l, m=-\infty}^{+\infty}{ }_{k} E_{l-1 / 2, m}^{x, \phi \phi} h_{k}(t) \phi_{l-1 / 2}(x) \phi_{m}(z) \\
& +\sum_{k, l, m=-\infty}^{+\infty} \sum_{r_{z}=0}^{+\infty} \sum_{p_{z}=0}^{2^{r_{z}}-1} E_{l-1 / 2, m}^{x, \phi \psi_{r_{z}, p_{z}}} h_{k}(t) \\
& \cdot \phi_{l-1 / 2}(x) \psi_{m, p_{z}}^{r_{z}}(z) \\
& +\sum_{k, l, m=-\infty}^{+\infty} \sum_{r_{x}=0}^{+\infty} \sum_{p_{x}=0}^{2^{r_{x}}-1}{ }_{k} E_{l-1 / 2, m}^{x, \psi_{r_{x}, p_{x}} \phi} h_{k}(t) \\
& \text { - } \psi_{l-1 / 2, p_{x}}^{r_{x}}(x) \phi_{m}(z) \\
& +\sum_{k, l, m=-\infty}^{+\infty} \sum_{r_{x}, r_{z}=0}^{+\infty} \sum_{p_{x}, p_{z}=0}^{2^{r_{x}}, r_{z}-1}{ }_{k} E_{l-1 / 2, m}^{x, \psi_{r_{x}, p_{x}} \psi_{r_{z}, p_{z}}} \\
& \text { - } h_{k}(t) \psi_{l-1 / 2, p_{x}}^{r_{x}}(x) \psi_{m, p_{z}}^{r_{z}}(z)
\end{aligned}
$$

$$
\begin{aligned}
& E_{z}(x, z, t) \\
& =\sum_{k, l, m=-\infty}^{+\infty}{ }_{k} E_{l, m-1 / 2}^{z, \phi \phi} h_{k}(t) \phi_{l}(x) \phi_{m-1 / 2}(z) \\
& +\sum_{k, l, m=-\infty}^{+\infty} \sum_{r_{z}=0}^{+\infty} \sum_{p_{z}=0}^{2^{r_{z}}-1}{ }_{k} E_{l, m-1 / 2}^{z, \phi \psi_{r_{z}, p_{z}}} h_{k}(t) \\
& \text { - } \phi_{l}(x) \psi_{m-1 / 2, p_{z}}^{r_{z}}(z) \\
& +\sum_{k, l, m=-\infty}^{+\infty} \sum_{r_{x}=0}^{+\infty} \sum_{p_{x}=0}^{2^{r_{x}}-1}{ }_{k} E_{l, m-1 / 2}^{z, \psi_{r_{x}, p_{x}} \phi} h_{k}(t) \\
& \text { - } \psi_{l, p_{x}}^{r_{2}}(x) \phi_{m-1 / 2}(z) \\
& +\sum_{k, l, m=-\infty}^{+\infty} \sum_{r_{x}, r_{z}=0}^{+\infty} \sum_{p_{x}, p_{z}=0}^{2^{r_{x}, r_{z}}-1} k E_{l, m-1 / 2}^{z, \psi_{r_{x}, p_{x}} \psi_{r_{z}, p_{z}}} h_{k}(t) \\
& \text { - } \psi_{l, p_{x}}^{r_{x}}(x) \psi_{m-1 / 2, p_{z}}^{r_{z}}(z) \\
& H_{y}(x, z, t) \\
& =\sum_{k, l, m=-\infty}^{+\infty} k+1 / 2 H_{l-1 / 2, m-1 / 2}^{y, \phi \phi} h_{k+1 / 2}(t) \\
& \text { - } \phi_{l-1 / 2}(x) \phi_{m-1 / 2}(z) \\
& +\sum_{k, l, m=-\infty}^{+\infty} \sum_{r_{z}=0}^{+\infty} \sum_{p_{z}=0}^{2^{r_{z}}-1} k+1 / 2 H_{l-1 / 2, m-1 / 2}^{y, \phi \psi_{r_{z}, p_{z}}} h_{k+1 / 2}(t) \\
& \cdot \phi_{l-1 / 2}(x) \psi_{m-1 / 2, p_{z}}^{r_{z}}(z) \\
& +\sum_{k, l, m=-\infty}^{+\infty} \sum_{r_{x}=0}^{+\infty} \sum_{p_{x}=0}^{2^{r_{x}}-1} k+1 / 2 H_{l-1 / 2, m-1 / 2}^{y, \psi_{r_{x}}, p_{x} \phi} h_{k+1 / 2}(t) \\
& \text { - } \psi_{l-1 / 2, p_{x}}^{r_{x}}(x) \phi_{m-1 / 2}(z) \\
& +\sum_{k, l, m=-\infty}^{+\infty} \sum_{r_{x}, r_{z}=0}^{+\infty} \sum_{p_{x}, p_{z}=0}^{2^{r_{x}}, r_{z}}-1 \quad k+1 / 2 H_{l-1 / 2, m-1 / 2}^{y, \psi_{r_{x}}, p_{x} \psi_{r_{z}, p_{z}}} \\
& \text { - } h_{k+1 / 2}(t) \psi_{l-1 / 2, p_{x}}^{r_{x}}(x) \psi_{m-1 / 2, p_{z}}^{r_{z}}(z)
\end{aligned}
$$

where $\phi_{n}(x)=\phi((x / \Delta x)-n)$ and $\psi_{n, p}^{r}(x)=2^{r / 2} \psi_{0}$ $\left(2^{r}[(x / \Delta x)-n]-p\right)$ represent the Battle-Lemarie scaling and $r$-resolution wavelet function, respectively. The expressions of the scaling and the zero-resolution wavelet in the spectral domain are given in (9) and (10). Since higher resolutions of wavelets are shifted and dilated versions of the zero resolution, their domain will be a fraction of that of the zero-resolution wavelet; thus, there is going to be more than one higher resolution wavelet coefficient for each MRTD cell. Specifically, for the arbitrary $r$ resolution and for the $n$ cell to the $x$-direction, there exist $2^{r}$ wavelet coefficients located at $x / \Delta x=n+\left(p / 2^{r+1}\right), p=0, \ldots, 2^{r}-1$. This is the reason for the summation of the $p$ terms for each resolution $r$ in the expansion of (14). ${ }_{k} E_{l, m}^{\kappa, \mu \nu}$ and ${ }_{k+1 / 2} H_{l, m}^{\kappa, \mu \nu}$ with $\kappa=x, y, z$ and $\mu, \nu=\phi, \psi$ are the coefficients for the field expansions in terms of scaling and wavelet functions. Schemes based on a displacement of $H$ and $E$ by $(1 / 2)^{(r x, r z)+2}$ instead of $1 / 2$ could provide slightly improved numerical dispersion and stability characteristics. Nevertheless, for simplicity reasons, the Yee's convention is used for the derivation of the MRTD equations. The indexes $l, m$ and $k$ are the discrete space and time indexes related to the space and time coordinates via 
$x=l \Delta x, z=m \Delta z$ and $t=k \Delta t$, where $\Delta x, \Delta z$ are the space discretization intervals in the $x$ - and $z$-directions and $\Delta t$ is the time discretization interval. For an accuracy of $0.1 \%$, the above summations are truncated to a finite number of terms determined by the dispersion and stability requirements (typically their maximum value is between 22-26). The time-domain expansion function $h_{k}(t)$ is defined as

$$
h_{k}(t)=h\left(\frac{t}{\Delta t}-k\right)
$$

with the rectangular pulse function

$$
h(t)= \begin{cases}1, & \text { for }|x|<1 / 2 \\ 1 / 2, & \text { for }|x|=1 / 2 \\ 0, & \text { for }|x|>1 / 2\end{cases}
$$

The magnetic-field components are shifted by half a discretization interval in the space and time domains with respect to the electric-field components (leap-frog).

Upon inserting the field expansions, Maxwell's equations are sampled using pulse functions as time-domain test functions and scaling/wavelet functions as space-domain test functions. For the sampling in the time domain, the following integrals are utilized:

$$
\int_{-\infty}^{+\infty} h_{k}(t) h_{k^{\prime}}(t) d t=\delta_{k, k^{\prime}} \Delta x
$$

and

$$
\int_{-\infty}^{+\infty} h_{k}(t) \frac{\partial h_{k^{\prime}+1 / 2}(t)}{\partial t} d t=\delta_{k, k^{\prime}}-\delta_{k, k^{\prime}+1}
$$

where $\delta_{k, k^{\prime}}$ is the Kroenecker symbol

$$
\delta_{k, k^{\prime}}= \begin{cases}1, & \text { for } k=k^{\prime} \\ 0, & \text { for } k \neq k^{\prime} .\end{cases}
$$

\section{B. Battle-Lemarie Expansion Basis}

Sampling in the space domain is obtained by use of the orthogonality relationships for the Battle-Lemarie scaling and wavelet functions [12]

$$
\begin{gathered}
\int_{-\infty}^{+\infty} \phi_{m}(x) \phi_{m^{\prime}}(x) d x=\delta_{m, m^{\prime}} \Delta x \\
\int_{-\infty}^{+\infty} \phi_{m}(x) \psi_{m^{\prime}, p^{\prime}}^{r}(x) d x=0 \quad \forall r, p
\end{gathered}
$$

and

$$
\int_{-\infty}^{+\infty} \psi_{m, p}^{r}(x) \psi_{m^{\prime}, p^{\prime}}^{r^{\prime}}(x) d x=\delta_{r, r^{\prime}} \delta_{m, m^{\prime}} \delta_{p, p^{\prime}} \Delta x .
$$

The integrals containing derivatives can be approximated by the following expressions:

$$
\int_{-\infty}^{+\infty} \phi_{m}(x) \frac{\partial \phi_{m^{\prime}+1 / 2}(x)}{\partial x} d x \approx \sum_{i=-n_{a}}^{n_{a}-1} a(i) \delta_{m+i, m^{\prime}}
$$

with

$$
a(i)=\frac{1}{\pi} \int_{0}^{\infty}|\hat{\phi}(\xi)|^{2} \xi \sin [\xi(i+1 / 2)] d \xi
$$

and

$$
\int_{-\infty}^{+\infty} \phi_{m}(x) \frac{\partial \psi_{m^{\prime}+1 / 2, p}^{r}(x)}{\partial x} d x \approx \sum_{i=-n_{d, r, 1}}^{n_{d, r, 2}} d_{r}(i, p) \delta_{m+i, m^{\prime}}
$$

with

$$
\begin{aligned}
& d_{r}(i, p)=\frac{1}{\pi} \int_{0}^{\infty} 2^{-r / 2} \hat{\phi}_{m}(\xi)\left|\hat{\psi_{0}}\left(\xi / 2^{r}\right)\right| \xi \\
& \cdot \sin \left[\xi\left(i+0.5+p / 2^{r}+1 / 2^{r+1}\right)\right] d \xi
\end{aligned}
$$

and

$$
\int_{-\infty}^{+\infty} \psi_{m, p}^{r}(x) \frac{\partial \phi_{m^{\prime}+1 / 2}(x)}{\partial x} d x \approx \sum_{i=-n_{c, r, 1}}^{n_{c, r, 2}} c_{r}(i, p) \delta_{m+i, m^{\prime}}
$$

with

$$
\begin{aligned}
& c_{r}(i, p)=\frac{1}{\pi} \int_{0}^{\infty} 2^{-r / 2} \hat{\phi}_{m}(\xi)\left|\hat{\psi}_{0}\left(\xi / 2^{r}\right)\right| \xi \\
& \cdot \sin \left[\xi\left(i+0.5-p / 2^{r}-1 / 2^{r+1}\right)\right] d \xi
\end{aligned}
$$

and

$$
\begin{aligned}
\int_{-\infty}^{+\infty} \psi_{m, p_{1}}^{r_{1}}(x) & \frac{\partial \psi_{m^{\prime}+1 / 2, p_{2}}^{r_{2}}(x)}{\partial x} d x \\
& \approx \sum_{i=-n_{b, r_{1}, r_{2}, 1}}^{n_{b, r_{1}, r_{2}, 2}} b_{r_{1}, r_{2}}\left(i, p_{1}, p_{2}\right) \delta_{m+i, m^{\prime}}
\end{aligned}
$$

with

$$
\begin{aligned}
b_{r_{1}, r_{2}}\left(i, p_{1}, p_{2}\right) & \\
=\int_{0}^{\infty} & \left|\hat{\psi}_{0}\left(\xi / 2^{r_{1}}\right)\right|\left|\hat{\psi}_{0}\left(\xi / 2^{r_{2}}\right)\right| \xi \\
\cdot \sin & {\left[\xi \left(i+1 / 2+p_{2} / 2^{r_{2}}-p_{1} / 2^{r_{1}}+1 / 2^{r_{2}+1}\right.\right.} \\
& \left.\left.\quad-1 / 2^{r_{1}+1}\right)\right] d \xi .
\end{aligned}
$$

For the remainder of this section, an expansion only in a series of scaling and zero-resolution wavelet functions will be considered. Hints for the enhancement of additional wavelet resolutions will be presented where needed in the following sections. Since for the zero resolution $(r=0)$, there is only one wavelet coefficient per cell $(p=0)$, the $p$ symbols will be omitted from the definition of the $b, c, d$ coefficients, which will be given by

$$
\int_{-\infty}^{+\infty} \psi_{m}^{0}(x) \frac{\partial \phi_{m^{\prime}+1 / 2}(x)}{\partial x} d x \approx \sum_{i=-n_{c, 0,1}}^{n_{c, 0,2}} c_{0}(i) \delta_{m+i, m^{\prime}}
$$

with

$$
c_{0}(i)=\frac{1}{\pi} \int_{0}^{\infty} \hat{\phi}_{m}(\xi)\left|\hat{\psi}_{0}(\xi)\right| \xi \sin [\xi i] d \xi
$$

and

$$
\int_{-\infty}^{+\infty} \phi_{m}(x) \frac{\partial \psi_{m^{\prime}+1 / 2}^{0}(x)}{\partial x} d x \approx \sum_{i=-n_{d, 0,1}}^{n_{d, 0,2}} d_{0}(i) \delta_{m+i, m^{\prime}}
$$


TABLE I

COEFFICIENTS $a(i), b_{0}(i), c_{0}(i)$

\begin{tabular}{c|c|c|c}
\hline $\mathrm{i}$ & $a(i)$ & $b_{0}(i)$ & $c_{0}(i)$ \\
\hline 0 & 1.29161604157839 & 2.47253977327429 & 0. \\
1 & -0.155978843323672 & 0.9562282774123074 & $-4.659725793402785 \mathrm{E}-02$ \\
2 & $5.9606303324687290 \mathrm{E}-02$ & 0.1660591600788887 & $5.453939813583327 \mathrm{E}-02$ \\
3 & $-2.929157759806890 \mathrm{E}-02$ & $9.392437777679437 \mathrm{E}-02$ & $-3.699957746974982 \mathrm{E}-02$ \\
4 & $1.5362399457426780 \mathrm{E}-02$ & $3.141444475216036 \mathrm{E}-03$ & $2.057449098775452 \mathrm{E}-02$ \\
5 & $-8.184462325283712 \mathrm{E}-03$ & $1.349356908709108 \mathrm{E}-02$ & $-1.115303180864957 \mathrm{E}-02$ \\
6 & $4.3757585552354830 \mathrm{E}-03$ & $-2.858941810094752 \mathrm{E}-03$ & $5.976877725279031 \mathrm{E}-03$ \\
7 & $-2.342365356649461 \mathrm{E}-03$ & $2.778680514115529 \mathrm{E}-03$ & $-3.202621363952005 \mathrm{E}-03$ \\
8 & $1.252877717042020 \mathrm{E}-03$ & $-1.129446167303586 \mathrm{E}-03$ & $1.714086849566890 \mathrm{E}-03$ \\
9 & $-6.716635068590737 \mathrm{E}-04$ & $7.071507309377701 \mathrm{E}-04$ & $-9.176508438494196 \mathrm{E}-04$ \\
10 & $3.583506907489797 \mathrm{E}-04$ & $-3.491267305845643 \mathrm{E}-04$ & $4.911754748072018 \mathrm{E}-04$ \\
11 & $-1.931321684715780 \mathrm{E}-04$ & $1.952711419194906 \mathrm{E}-04$ & $-2.629253013538502 \mathrm{E}-04$ \\
12 & $1.019327767057869 \mathrm{E}-04$ & $-1.021304423384722 \mathrm{E}-04$ & $1.407386855875626 \mathrm{E}-04$ \\
13 & $-5.613943183518454 \mathrm{E}-05$ & $5.531259273864269 \mathrm{E}-05$ & $-7.533840689573666 \mathrm{E}-05$ \\
14 & $2.834596805928539 \mathrm{E}-05$ & $-2.947330468694831 \mathrm{E}-05$ & $4.033146235099674 \mathrm{E}-05$ \\
15 & $-1.700348604873522 \mathrm{E}-05$ & $1.572110653438641 \mathrm{E}-05$ & $-2.159462850665844 \mathrm{E}-05$ \\
\hline
\end{tabular}

with

$$
d_{0}(i)=\int_{0}^{\infty} \hat{\phi}_{m}(\xi)\left|\hat{\psi}_{0}(\xi)\right| \xi \sin (\xi i+1) d \xi=c_{0}(i+1) .
$$

Observing the similar form of (24)-(26), (31) can be written as

$$
\begin{aligned}
\int_{-\infty}^{+\infty} \phi_{m}(x) \frac{\partial \psi_{m^{\prime}+1 / 2}^{0}(x)}{\partial x} d x & \sum_{i=-n_{c, 0,1}-1}^{n_{c, 0,2}-1} c_{0}(i+1) \delta_{m+i, m^{\prime}}
\end{aligned}
$$

Also,

$$
\int_{-\infty}^{+\infty} \psi_{m}^{0}(x) \frac{\partial \psi_{m^{\prime}+1 / 2}^{0}(x)}{\partial x} d x \approx \sum_{i=-n_{b}, 0,0,1}^{n_{b, 0,0,2}} b_{0}(i) \delta_{m+i, m^{\prime}}
$$

with

$$
b_{0}(i)=b_{0,0}(i)=\int_{0}^{\infty}\left|\hat{\psi}_{0}(\xi)\right|^{2} \xi \sin [\xi(i+1 / 2)] d \xi
$$

with $a(i), b_{0}(i), c_{0}(i)$ given in Table I [4]. Due to symmetries in the integrals for the zero resolution, the coefficients satisfy the conditions $a(-1-i)=-a(i), b_{0}(-1-i)=-b_{0}(i)$, and $c_{0}(-i)=-c_{0}(i)$ for $i<0$. Hence, the stencil lengths have to be $n_{b, 0,0,1}=n_{b, 0,0,2}-1=n_{b}$ and $n_{c, 0,1}=n_{c, 0,2}=n_{c}$. These conditions are not general and do not hold for any other arbitrary resolution. The stencil size is determined by the dispersion requirements. It has to be noted that the Battle-Lemarie scaling function has exponential decay; thus, the coefficients $a(i)$ for $i>12$ are not zero, but their value is negligible $\left(\leq 10^{-4}\right)$.

After applying the Galerkin technique to (11)-(13), the following MRTD equations are derived:

$$
\begin{aligned}
& \frac{{ }_{k+1} E_{l-1 / 2, m}^{x, \phi \phi}-{ }_{k} E_{l-1 / 2, m}^{x, \phi \phi}}{\Delta t} \\
& =-\frac{1}{\epsilon \Delta z}\left(\sum_{m^{\prime}=-n_{a}}^{n_{a}-1} a\left(m^{\prime}\right)_{k+1 / 2} H_{l-1 / 2, m+m^{\prime}+1-1 / 2}^{y, \phi \phi}\right. \\
& \left.+\sum_{m^{\prime}=-n_{c}}^{n_{c}} c_{0}\left(m^{\prime}\right)_{k+1 / 2} H_{l-1 / 2, m+m^{\prime}-1 / 2}^{y, \phi \psi_{0}}\right) \\
& \frac{{ }_{k+1} E_{l-1 / 2, m}^{x, \psi_{0} \phi}-{ }_{k} E_{l-1 / 2, m}^{x, \psi_{0} \phi}}{\Delta t} \\
& =-\frac{1}{\epsilon \Delta z}\left(\sum_{m^{\prime}=-n_{a}}^{n_{a}-1} a\left(m^{\prime}\right)_{k+1 / 2} H_{l-1 / 2, m+m^{\prime}+1-1 / 2}^{z, \psi_{0} \phi}\right. \\
& \left.+\sum_{m^{\prime}=-n_{c}}^{n_{c}} c_{0}\left(m^{\prime}\right)_{k+1 / 2} H_{l-1 / 2, m+m^{\prime}-1 / 2}^{y, \psi_{0} \psi_{0}}\right)
\end{aligned}
$$




$$
\begin{aligned}
& \begin{array}{l}
\frac{k_{l+1} E_{l-1 / 2, m}^{x, \phi \psi_{0}}-{ }_{k} E_{l-1 / 2, m}^{x, \phi \psi_{0}}}{\Delta t} \\
=-\frac{1}{\epsilon \Delta z}\left(\sum_{m^{\prime}=-n_{c}}^{n_{c}} c_{0}\left(m^{\prime}\right)_{k+1 / 2} H_{l-1 / 2, m+m^{\prime}+1-1 / 2}^{y, \phi \phi}\right. \\
\left.\quad+\sum_{m^{\prime}=-n_{b}}^{n_{b}-1} b_{0}\left(m^{\prime}\right)_{k+1 / 2} H_{l-1 / 2, m+m^{\prime}+1-1 / 2}^{y, \phi \psi_{0}}\right)
\end{array} \\
& \frac{{ }_{k+1} E_{l-1 / 2, m}^{x, \psi_{0} \psi_{0}}-{ }_{k} E_{l-1 / 2, m}^{x, \psi_{0} \psi_{0}}}{\Delta t} \\
& =-\frac{1}{\epsilon \Delta z}\left(\sum_{m^{\prime}=-n_{c}}^{n_{c}} c_{0}\left(m^{\prime}\right)_{k+1 / 2} H_{l-1 / 2, m+m^{\prime}+1-1 / 2}^{y, \psi_{0} \phi}\right. \\
& \left.+\sum_{m^{\prime}=-n_{b}}^{n_{b}-1} b_{0}\left(m^{\prime}\right)_{k+1 / 2} H_{l-1 / 2, m+m^{\prime}+1-1 / 2}^{y, \psi_{0} \psi_{0}}\right) \\
& \frac{{ }_{k+1} E_{l, m-1 / 2}^{z, \phi \phi}-{ }_{k} E_{l, m-1 / 2}^{z, \phi \phi}}{\Delta t} \\
& =\frac{1}{\epsilon \Delta x}\left(\sum_{l^{\prime}=-n_{a}}^{n_{a}-1} a\left(l^{\prime}\right)_{k+1 / 2} H_{l+l^{\prime}+1-1 / 2, m-1 / 2}^{y, \phi \phi}\right. \\
& \left.+\sum_{l^{\prime}=-n_{c}}^{n_{c}} c_{0}\left(l^{\prime}\right)_{k+1 / 2} H_{l+l^{\prime}-1 / 2, m-1 / 2}^{y, \psi_{0} \phi}\right) \\
& \frac{{ }_{k+1} E_{l, m-1 / 2}^{z, \phi \psi_{0}}-{ }_{k} E_{l, m-1 / 2}^{z, \phi \psi_{1} 0}}{\Delta t} \\
& =\frac{1}{\epsilon \Delta x}\left(\sum_{l^{\prime}=-n_{a}}^{n_{a}-1} a\left(l^{\prime}\right)_{k+1 / 2} H_{l+l^{\prime}+1-1 / 2, m-1 / 2}^{y, \phi \psi_{0}}\right. \\
& \left.+\sum_{l^{\prime}=-n_{c}}^{n_{c}} c_{0}\left(l^{\prime}\right)_{k+1 / 2} H_{l+l^{\prime}-1 / 2, m-1 / 2}^{y, \psi_{0} \psi_{0}}\right) \\
& \frac{{ }_{k+1} E_{l, m-1 / 2}^{z, \psi_{0} \phi}-{ }_{k} E_{l, m-1 / 2}^{z, \psi_{0} \phi}}{\Delta t} \\
& =\frac{1}{\epsilon \Delta x}\left(\sum_{l^{\prime}=-n_{c}}^{n_{c}} c_{0}\left(l^{\prime}\right)_{k+1 / 2} H_{l+l^{\prime}+1-1 / 2, m-1 / 2}^{y, \phi \phi}\right. \\
& \left.+\sum_{l^{\prime}=-n_{b}}^{n_{b}-1} b_{0}\left(l^{\prime}\right)_{k+1 / 2} H_{l+l^{\prime}+1-1 / 2, m-1 / 2}^{y, \psi_{0} \phi}\right) \\
& \frac{{ }_{k+1} E_{l, m-1 / 2}^{z, \psi_{0} \psi_{0}}-{ }_{k} E_{l, m-1 / 2}^{z, \psi_{0} \psi_{0}}}{\Delta t} \\
& =\frac{1}{\epsilon \Delta x}\left(\sum_{l^{\prime}=-n_{c}}^{n_{c}} c_{0}\left(l^{\prime}\right)_{k+1 / 2} H_{l+l^{\prime}+1-1 / 2, m-1 / 2}^{y, \phi \psi_{0}}\right. \\
& \left.+\sum_{l^{\prime}=-n_{b}}^{n_{b}-1} b_{0}\left(l^{\prime}\right)_{k+1 / 2} H_{l+l^{\prime}+1-1 / 2, m-1 / 2}^{y, \psi_{0} \psi_{0}}\right) \\
& \frac{{ }_{k+1 / 2} H_{l-1 / 2, m-1 / 2}^{y, \phi \phi}-{ }_{k-1 / 2} H_{l-1 / 2, m-1 / 2}^{y, \phi \phi}}{\Delta t} \\
& =\frac{1}{\mu}\left[\frac { 1 } { \Delta x } \left(\sum_{l^{\prime}=-n_{a}}^{n_{a}-1} a\left(l^{\prime}\right)_{k} E_{l+l^{\prime}, m-1 / 2}^{z, \phi \phi}\right.\right. \\
& \left.+\sum_{l^{\prime}=-n_{c}}^{n_{c}} c_{0}\left(l^{\prime}\right)_{k} E_{l+l^{\prime}-1, m-1 / 2}^{z, \psi_{0} \phi}\right)
\end{aligned}
$$

$$
\begin{aligned}
& -\frac{1}{\Delta z}\left(\sum_{m^{\prime}=-n_{a}}^{n_{a}-1} a\left(m^{\prime}\right)_{k} E_{l-1 / 2, m+m^{\prime}}^{x, \phi \phi}\right. \\
& \left.\left.+\sum_{m^{\prime}=-n_{c}}^{n_{c}} c_{0}\left(m^{\prime}\right)_{k} E_{l-1 / 2, m+m^{\prime}-1}^{x, \phi \psi_{0}}\right)\right] \\
& \frac{k+1 / 2}{k H_{l-1 / 2, m-1 / 2}^{y, \psi_{0} \phi}-{ }_{k-1 / 2} H_{l-1 / 2, m-1 / 2}^{y, \psi_{0} \phi}} \\
& =\frac{1}{\mu}\left[\frac { 1 } { \Delta x } \left(\sum_{l^{\prime}=-n_{c}}^{n_{c}} c_{0}\left(l^{\prime}\right)_{k} E_{l+l^{\prime}, m-1 / 2}^{z, \phi \phi}\right.\right. \\
& \left.+\sum_{l^{\prime}=-n_{b}}^{n_{b}-1} b_{0}\left(l^{\prime}\right)_{k} E_{l+l^{\prime}, m-1 / 2}^{z, \psi_{0} \phi}\right) \\
& -\frac{1}{\Delta z}\left(\sum_{m^{\prime}=-n_{a}}^{n_{a}-1} a\left(m^{\prime}\right)_{k} E_{l-1 / 2, m+m^{\prime}}^{x, \psi_{0} \phi}\right. \\
& \left.\left.+\sum_{m^{\prime}=-n_{c}}^{n_{c}} c_{0}\left(m^{\prime}\right)_{k} E_{l-1 / 2, m+m^{\prime}-1}^{x, \psi_{0} \psi_{0}}\right)\right]
\end{aligned}
$$

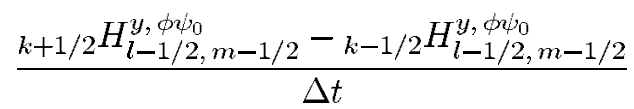

$$
\begin{aligned}
& =\frac{1}{\mu}\left[\frac { 1 } { \Delta x } \left(\sum_{l^{\prime}=-n_{a}}^{n_{a}-1} a\left(l^{\prime}\right)_{k} E_{l+l^{\prime}, m-1 / 2}^{z, \phi \psi_{0}}\right.\right. \\
& \left.+\sum_{l^{\prime}=-n_{c}}^{n_{c}} c_{0}\left(l^{\prime}\right)_{k} E_{l+l^{\prime}-1, m-1 / 2}^{z, \psi_{0} \psi_{0}}\right) \\
& -\frac{1}{\Delta z}\left(\sum_{m^{\prime}=-n_{c}}^{n_{c}} c_{0}\left(m^{\prime}\right)_{k} E_{l-1 / 2, m+m^{\prime}}^{x, \phi \phi}\right. \\
& \left.\left.+\sum_{m^{\prime}=-n_{b}}^{n_{b}-1} b_{0}\left(m^{\prime}\right)_{k} E_{l-1 / 2, m+m^{\prime}}^{x, \phi \psi_{0}}\right)\right]
\end{aligned}
$$

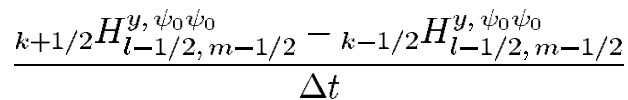

$$
\begin{aligned}
& =\frac{1}{\mu}\left[\frac { 1 } { \Delta x } \left(\sum_{l^{\prime}=-n_{c}}^{n_{c}} c_{0}\left(l^{\prime}\right)_{k} E_{l+l^{\prime}, m-1 / 2}^{z, \phi \psi_{0}}\right.\right. \\
& \left.+\sum_{l^{\prime}=-n_{b}}^{n_{b}-1} b_{0}\left(l^{\prime}\right)_{k} E_{l+l^{\prime}, m-1 / 2}^{z, \psi_{0} \psi_{0}}\right) \\
& -\frac{1}{\Delta z}\left(\sum_{m^{\prime}=-n_{c}}^{n_{c}} c_{0}\left(m^{\prime}\right)_{k} E_{l-1 / 2, m+m^{\prime}}^{x, \psi_{0} \phi}\right. \\
& \left.\left.+\sum_{m^{\prime}=-n_{b}}^{n_{b}-1} b_{0}\left(m^{\prime}\right)_{k} E_{l-1 / 2, m+m^{\prime}}^{x, \psi_{0} \psi_{0}}\right)\right] .
\end{aligned}
$$

The values of the stencil lengths $n_{a}, n_{b}, n_{c}$ depends on the accuracy and dispersion requirements. The discretization cell is similar to the conventional FDTD cell (Fig. 5).

In [18], the stability limit for the 2-D MRTD scheme based only on the scaling functions' expansion (S-MRTD) was found to be given by

$$
\Delta t \leq \frac{1}{c\left(\sum_{i^{\prime}=0}^{n_{a}-1}\left|a\left(i^{\prime}\right)\right|\right) \sqrt{\frac{1}{(\Delta x)^{2}}+\frac{1}{(\Delta y)^{2}}}}
$$




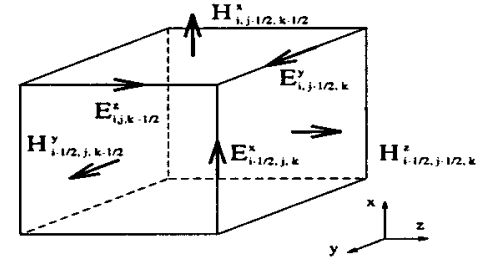

Fig. 5. MRTD discretization cell in 3-D.

where $\Delta x, \Delta y$ are the cell dimensions and $c=1 / \sqrt{\mu \epsilon}$ is the velocity of the light in the modeled medium.

For $\Delta x=\Delta y=\Delta$, the above stability criterion gives

$$
\Delta t_{S-\mathrm{MRTD}} \leq \frac{\Delta}{c \sqrt{2} \sum_{i^{\prime}=0}^{n_{a}-1}\left|a\left(i^{\prime}\right)\right|}=s_{S S} \frac{\Delta}{c \sqrt{2}} .
$$

It is known [2] that

$$
\Delta t_{\mathrm{FDTD}} \leq \frac{1}{c \sqrt{\frac{1}{(\Delta x)^{2}}+\frac{1}{(\Delta y)^{2}}}}
$$

which gives for $\Delta x=\Delta y=\Delta$

$$
\Delta t_{\mathrm{FDTD}} \leq \frac{\Delta}{c \sqrt{2}}
$$

Equations (38)-(40) show that, for same discretization size, the upper bounds of the time steps of FDTD and S-MRTD are comparable and related through the factor $s$. The stability analysis can be generalized easily to three-dimensional (3-D). The new stability criteria can be derived by (38) and (40) by substituting the term $\sqrt{2}$ with $\sqrt{3}$.

More complicated expressions can be derived for the maximum allowable time step for schemes containing scaling and wavelet functions. For simplicity and without loss of generality, it is assumed that the stencil size is equal for all three summations $\left(n_{a}=n_{b}=n_{c}=n\right)$. The upper bound of the time step for the 2-D MRTD scheme with zero-resolution wavelets to the one ( $x$-direction) or two directions ( $x$ - and $y$-directions) for $\Delta x=\Delta y=\Delta$ is given by

$$
\Delta t_{W_{0} S \text {-MRTD, } \max } \approx s_{W_{0} S} \frac{\Delta}{c \sqrt{2}}
$$

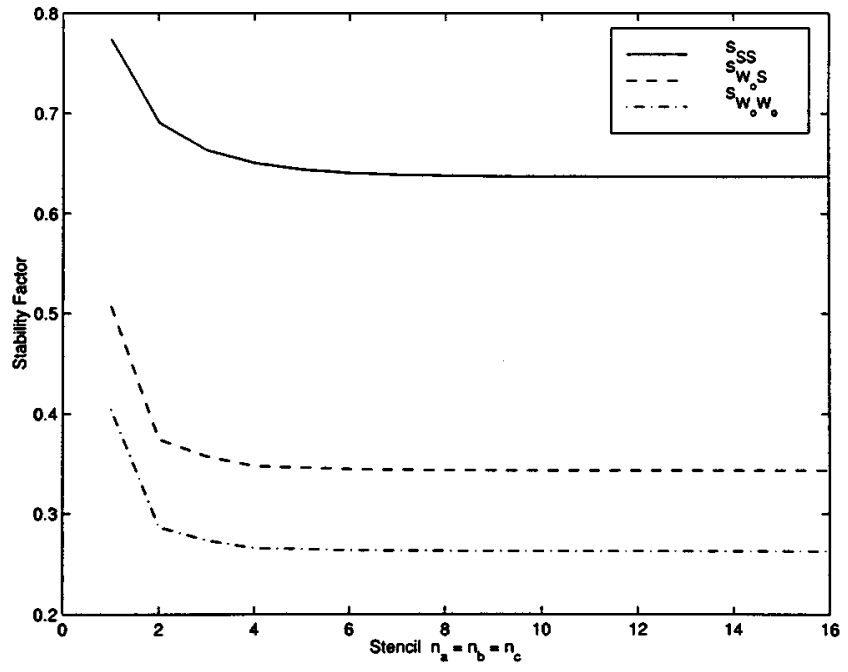

Fig. 6. Stability parameter $s$ for MRTD.

with the first equation shown at the bottom of this page, and

$$
\Delta t_{W_{0} W_{0}-\mathrm{MRTD}, \max } \approx s_{W_{0} W_{0}} \frac{\Delta}{c \sqrt{2}}
$$

with the second equation shown at the bottom of this page, where the notation

$$
\sum_{k^{\prime}}|x|=\sum_{k^{\prime}=0}^{n-1}\left|x\left(k^{\prime}\right)\right|
$$

has been used.

It can be observed that the upper bound of the time step depends on the stencil size $n_{a}, n_{b}, n_{c}$. This dependence is expressed through the coefficients $s_{S S}, s_{W_{0} S}, s_{W_{0} W_{0}}$, which decrease as the stencil size increases. Fig. 6 shows that $s_{S S}$ practically converges to the value 0.6371 after $n_{a} \geq 10$ and $s_{W_{0} S} \approx$ 0.3433 and $s_{W_{0} W_{0}} \approx 0.2625$ for $n_{a}=n_{b}=n_{c} \geq 10$. The expression of $s_{S S}$ can be easily derived by the expressions of $s_{W_{0} S}$ and $s_{W_{0} W_{0}}$ by zeroing out the effect of $b_{0}, c_{0}$. The above expressions can be generalized for $r_{\max }$ resolutions of wavelets by dividing the time-step expression by $2^{r_{\max }}$ due to the dilation of the $r_{\max }$ wavelet resolution.

2

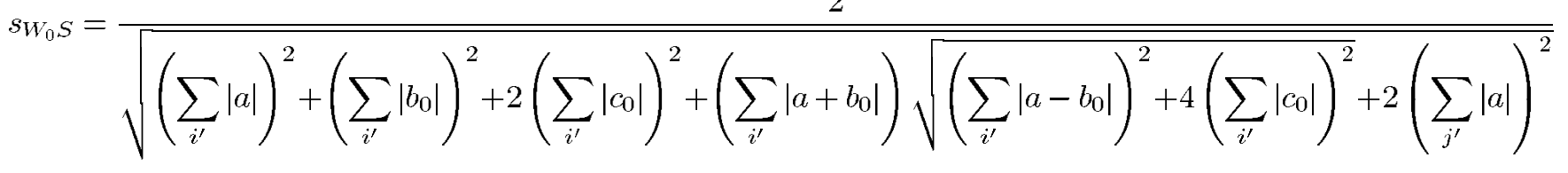

$$
s_{W_{0} W_{0}}=\frac{1}{\sqrt{\left(\sum_{i^{\prime}}|a|\right)^{2}+\left(\sum_{i^{\prime}}\left|b_{0}\right|\right)^{2}+2\left(\sum_{i^{\prime}}\left|c_{0}\right|\right)^{2}+\left(\sum_{i^{\prime}}\left|a+b_{0}\right|\right) \sqrt{\left(\sum_{i^{\prime}}\left|a-b_{0}\right|\right)^{2}+4\left(\sum_{i^{\prime}}\left|c_{0}\right|\right)^{2}}}}
$$




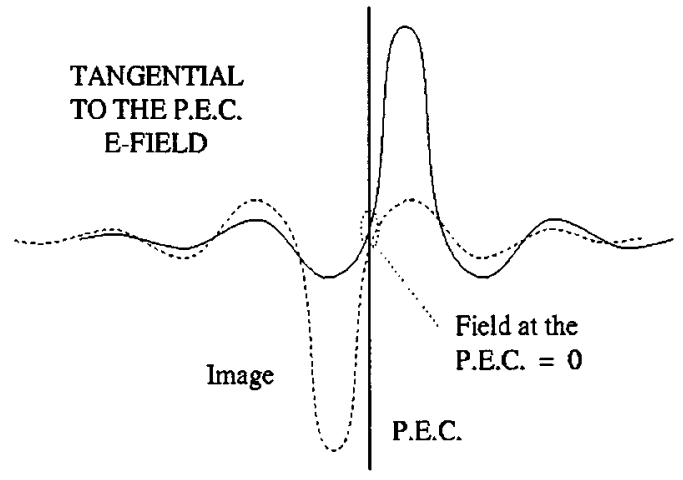

Fig. 7. Image theory application for tangential-to-PEC $E$-field.

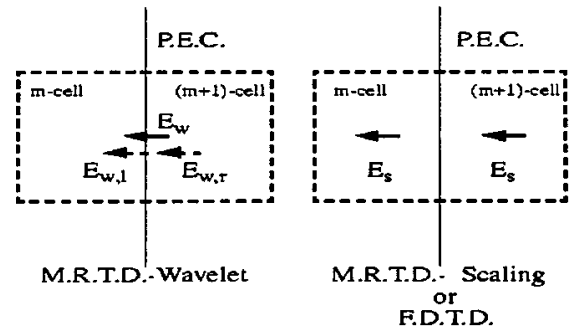

Fig. 8. Treatment of wavelet components of normal-to-PEC $E$-field.

In addition, the dispersion curves have demonstrated that cell sizes close to the Nyquist limit provide acceptable accuracy even for zero resolution of wavelets. These schemes have been proven to be equivalent to 22nd-order FDTD schemes [18]. On the contrary, conventional FDTD (second-order) simulations require much smaller cell sizes (close or below $\lambda / 10$ ).

1) Modeling of Hard Boundaries: Unlike the FDTD where the consistency with the image theory is implicit in the application of the boundary conditions, for MRTD schemes based on entire-domain functions, this theory must be applied explicitly in the locations of perfect electric conductors (PECs) or perfect magnetic conductors (PMCs). The total value of a field component at a specific cell is affected by a theoretically infinite-practically finite-number of neighboring cells due to the fact that the basis functions extend from $-\infty$ to $\infty$. Some of these neighbors may be located on the other side of the conductor. This effect is taken into consideration by applying the image theory (Fig. 7). In this way, the physical boundary condition of zeroing-out the $E$-field tangential to the PEC is automatically satisfied. For example, even symmetry is applied for the normal-to-PEC electric-field components and odd symmetry for the parallel-to-PEC. Image theory can be implemented automatically for an arbitrary number of hard boundaries.

The time-domain numerical techniques are modeling the real space by creating a discrete numerical grid. Sometimes this mesh does not coincide with the electrical one, and MRTD is one example. The enhancement of wavelets on MRTD requires a special treatment of the wavelet components of the normal-to-PEC electric field. Assuming a vertical PEC in Fig. 8, the electrical domains (I) and (II) are isolated from each other. That means that one wavelet component value of the normal electric field EXACTLY ON the PEC would create a nonphysical electrical coupling. Thus, Two wavelet components, one located infinitesimally on the left-hand side of the PEC and the other infinitesimally located on the right-hand side of the PEC, have to be defined in order to satisfy the electrical isolation condition. The $H$-field component that is parallel to the PEC has to be treated in a similar way. The rest components of the $E$ - and $H$-field have to be zeroed-out on both sides of the PEC, so one value is sufficient. In FDTD, the interleaved positioning of the field components on the Yee's cell (which are the same with the scaling functions components on the MRTD's cell) requires that the normal-to-PEC $E$-field component is located a half cell size away from the conductor. In this way, the definition of only one field component per cell is sufficient.

2) Modeling of Dielectric Interfaces: Due to the entire domain basis functions, $D$ of one cell is related to $E$ values all over the neighboring cells [19]. To circumvent this problem, the Curl $D$ equations have to replace the $\operatorname{Curl} E$ equations and then the $E$-coefficients have to be calculated from the $D$-coefficients in a mathematically correct way. After inserting the field expansions in Maxwell's equations, we sample them using pulse functions in time and scaling/wavelet functions in space domain.

As an example, sampling $\partial D_{x} / \partial t=-\partial H_{y} / \partial z$ in space and time, the following equations are obtained:

$$
\begin{aligned}
& \frac{k_{k+1} D_{l-1 / 2, m}^{x, \phi \phi}-{ }_{k} D_{l-1 / 2, m}^{x, \phi \phi}}{\Delta t} \\
& =-\frac{1}{\Delta z}\left(\sum_{m^{\prime}=-n_{a}}^{n_{a}-1} a\left(m^{\prime}\right)_{k+1 / 2} H_{l-1 / 2, m+m^{\prime}+1-1 / 2}^{y, \phi \phi}\right. \\
& \left.+\sum_{m^{\prime}=-n_{c}}^{n_{c}} c_{0}\left(m^{\prime}\right)_{k+1 / 2} H_{l-1 / 2, m+m^{\prime}-1 / 2}^{y, \phi \psi_{0}}\right) \\
& \frac{{ }_{k+1} D_{l-1 / 2, m}^{x, \psi_{0} \phi}-{ }_{k} D_{l-1 / 2, m}^{x, \psi_{0} \phi}}{\Delta t} \\
& =-\frac{1}{\Delta z}\left(\sum_{m^{\prime}=-n_{a}}^{n_{a}-1} a\left(m^{\prime}\right)_{k+1 / 2} H_{l-1 / 2, m+m^{\prime}+1-1 / 2}^{z, \psi_{0} \phi}\right. \\
& \left.+\sum_{m^{\prime}=-n_{c}}^{n_{c}} c_{0}\left(m^{\prime}\right)_{k+1 / 2} H_{l-1 / 2, m+m^{\prime}-1 / 2}^{y, \psi_{0} \psi_{0}}\right) \\
& { }_{k+1} D_{l-1 / 2, m}^{x, \phi \psi_{0}}-{ }_{k} D_{l-1 / 2, m}^{x, \phi \psi_{0}} \\
& =-\frac{1}{\Delta z}\left(\sum_{m^{\prime}=-n_{c}}^{n_{c}} c_{0}\left(m^{\prime}\right)_{k+1 / 2} H_{l-1 / 2, m+m^{\prime}+1-1 / 2}^{y, \phi \phi}\right. \\
& \left.+\sum_{m^{\prime}=-n_{b}}^{n_{b}-1} b_{0}\left(m^{\prime}\right)_{k+1 / 2} H_{l-1 / 2, m+m^{\prime}+1-1 / 2}^{y, \phi \psi_{0}}\right) \\
& \frac{{ }_{k+1} D_{l-1 / 2, m}^{x, \psi_{0} \psi_{0}}-{ }_{k} D_{l-1 / 2, m}^{x, \psi_{0} \psi_{0}}}{\Delta t} \\
& =-\frac{1}{\Delta z}\left(\sum_{m^{\prime}=-n_{c}}^{n_{c}} c_{0}\left(m^{\prime}\right)_{k+1 / 2} H_{l-1 / 2, m+m^{\prime}+1-1 / 2}^{y, \psi_{0} \phi}\right. \\
& \left.+\sum_{m^{\prime}=-n_{b}}^{n_{b}-1} b_{0}\left(m^{\prime}\right)_{k+1 / 2} H_{l-1 / 2, m+m^{\prime}+1-1 / 2}^{y, \psi_{0} \psi_{0}}\right)
\end{aligned}
$$


where ${ }_{k} D_{l, m}^{x, \zeta \xi}$ and ${ }_{k} H_{l, m}^{y, \zeta \xi}$ with $\zeta, \xi=\phi$ (scaling), $\psi_{o}$ (wavelets of zero resolution) are the coefficients for the electric- and magnetic-field expansions.

Starting from the constitutive relationship $D=\epsilon E$ for the total electric field at one mesh point and sampling the scaling and wavelet components, we reach the following equations for $D_{x}$ :

$$
\begin{aligned}
& { }_{k} D_{l, m}^{x, \phi \phi} \\
& =\sum_{l^{\prime}=-l_{\phi}}^{l_{\phi}}\left(\sum_{m^{\prime}=-l_{\phi}}^{l_{\phi}} c_{\phi \phi}^{\phi \phi, d}\left(l^{\prime}, m^{\prime}\right)_{k} E_{l+l^{\prime}, m+m^{\prime}}^{x, \phi \phi}\right. \\
& \left.+\sum_{m^{\prime}=-l_{\psi_{o}}}^{l_{\psi_{o}}} c_{\phi \psi_{o}}^{\phi \phi, d}\left(l^{\prime}, m^{\prime}\right)_{k} E_{l+l^{\prime}, m+m^{\prime}}^{x, \phi \psi_{o}}\right) \\
& +\sum_{l^{\prime}=-l_{\psi_{o}}}^{l_{\psi_{o}}}\left(\sum_{m^{\prime}=-l_{\phi}}^{l_{\phi}} c_{\psi_{o} \phi}^{\phi \phi, d}\left(l^{\prime}, m^{\prime}\right)_{k} E_{l+l^{\prime}, m+m^{\prime}}^{x, \psi_{o} \phi}\right. \\
& \left.+\sum_{m^{\prime}=-l_{\psi_{o}}}^{l_{\psi_{o}}} c_{\psi_{o} \psi_{o}}^{\phi \phi, d}\left(l^{\prime}, m^{\prime}\right)_{k} E_{l+l^{\prime}, m+m^{\prime}}^{x, \psi_{o} \psi_{o}}\right)
\end{aligned}
$$$$
{ }_{k} D_{l, m}^{x, \psi_{\circ}}
$$$$
=\sum_{l^{\prime}=-l_{\phi}}^{l_{\phi}}\left(\sum_{m^{\prime}=-l_{\phi}}^{l_{\phi}} c_{\phi \phi}^{\psi_{o} \phi, d}\left(l^{\prime}, m^{\prime}\right)_{k} E_{l+l^{\prime}, m+m^{\prime}}^{x, \phi \phi}\right.
$$$$
\left.+\sum_{m^{\prime}=-l_{\psi_{o}}}^{l_{\psi_{o}}} c_{\phi \psi_{o}}^{\psi_{o} \phi, d}\left(l^{\prime}, m^{\prime}\right)_{k} E_{l+l^{\prime}, m+m^{\prime}}^{x, \phi \psi_{o}}\right)
$$$$
+\sum_{l^{\prime}=-l_{\psi_{o}}}^{l_{\psi_{o}}}\left(\sum_{m^{\prime}=-l_{\phi}}^{l_{\phi}} c_{\psi_{o} \phi}^{\psi_{o} \phi, d}\left(l^{\prime}, m^{\prime}\right)_{k} E_{l+l^{\prime}, m+m^{\prime}}^{x, \psi_{o} \phi}\right.
$$$$
\left.+\sum_{m^{\prime}=-l_{\psi_{o}}}^{l_{\psi_{o}}} c_{\psi_{o} \psi_{o}}^{\psi_{o} \phi, d}\left(l^{\prime}, m^{\prime}\right)_{k} E_{l+l^{\prime}, m+m^{\prime}}^{x, \psi_{o}}\right)
$$

${ }_{k} D_{l, m}^{x, \phi \psi_{o}}$

$$
\begin{aligned}
& =\sum_{l^{\prime}=-l_{\phi}}^{l_{\phi}}\left(\sum_{m^{\prime}=-l_{\phi}}^{l_{\phi}} c_{\phi \phi}^{\phi \psi_{o}, d}\left(l^{\prime}, m^{\prime}\right)_{k} E_{l+l^{\prime}, m+m^{\prime}}^{x, \phi \phi}\right. \\
& \left.+\sum_{m^{\prime}=-l_{\psi_{o}}}^{l_{\psi_{o}}} c_{\phi \psi_{o}}^{\phi \psi_{o}, d}\left(l^{\prime}, m^{\prime}\right)_{k} E_{l+l^{\prime}, m+m^{\prime}}^{x, \phi \psi_{o}}\right) \\
& +\sum_{l^{\prime}=-l_{\psi_{o}}}^{l_{\psi_{o}}}\left(\sum_{m^{\prime}=-l_{\phi}}^{l_{\phi}} c_{\psi_{o} \phi}^{\phi \psi_{o}, d}\left(l^{\prime}, m^{\prime}\right)_{k} E_{l+l^{\prime}, m+m^{\prime}}^{x, \psi_{o} \phi}\right. \\
& \left.+\sum_{m^{\prime}=-l_{\psi_{o}}}^{l_{\psi_{o}}} c_{\psi_{o} \psi_{o}}^{\phi \psi_{o}, d}\left(l^{\prime}, m^{\prime}\right)_{k} E_{l+l^{\prime}, m+m^{\prime}}^{x, \psi_{o} \psi_{o}}\right)
\end{aligned}
$$

$$
\begin{aligned}
{ }_{k} D_{l, m}^{x, \psi_{o} \psi_{o}} & \sum_{l^{\prime}=-l_{\phi}}^{l_{\phi}}\left(\sum_{m^{\prime}=-l_{\phi^{\prime}}}^{l_{\phi}} c_{\phi \phi}^{\psi_{o} \psi_{o}, d}\left(l^{\prime}, m^{\prime}\right)_{k} E_{l+l^{\prime}, m+m^{\prime}}^{x, \phi \phi}\right. \\
& \left.+\sum_{m^{\prime}=-l_{\psi_{o}}}^{l_{\psi_{o}}} c_{\phi \psi_{o}}^{\psi_{o} \psi_{o}, d}\left(l^{\prime}, m^{\prime}\right)_{k} E_{l+l^{\prime}, m+m^{\prime}}^{x, \phi \psi_{o}}\right)
\end{aligned}
$$

$$
\begin{aligned}
+\sum_{l^{\prime}=-l_{\psi_{o}}}^{l_{\psi_{o}}} & \left(\sum_{m^{\prime}=-l_{\phi}}^{l_{\phi}} c_{\psi_{o} \phi}^{\psi_{o} \psi_{o}, d}\left(l^{\prime}, m^{\prime}\right)_{k} E_{l+l^{\prime}, m+m^{\prime}}^{x, \psi_{o} \phi}\right. \\
& \left.+\sum_{m^{\prime}=-l_{\psi_{o}}}^{l_{\psi_{o}}} c_{\psi_{o} \psi_{o}}^{\psi_{o} \psi_{o}, d}\left(l^{\prime}, m^{\prime}\right)_{k} E_{l+l^{\prime}, m+m^{\prime}}^{x, \psi_{o} \psi_{o}}\right)
\end{aligned}
$$

where

$$
\begin{array}{r}
c_{\zeta_{2} \xi_{2}}^{\zeta_{1} \xi_{1}, d}\left(l^{\prime}, m^{\prime}\right)=\int_{-\infty}^{\infty} \int_{-\infty}^{\infty} \epsilon(x, z) \zeta_{1, l}(x) \xi_{2, l+l^{\prime}}(x) \\
\cdot \xi_{1, m}(z) \xi_{2, m+m^{\prime}}(z) d z d x
\end{array}
$$

and $\zeta_{1}, \zeta_{2}, \xi_{1}, \xi_{2}=\phi$ (scaling), $\psi_{o}$ (wavelets of zero resolution). Applying these equations for all neighboring cells that have nonnegligible values for at least one $c_{\zeta_{2} \xi_{2}}^{\zeta_{1} \xi_{1}, d}\left(l^{\prime}, m^{\prime}\right)$ coefficient, the following compact form is derived:

$$
[\bar{D}]=[\overline{\bar{\epsilon}}][\bar{E}]
$$

For geometries with dielectrics varying from air $\left(\epsilon_{r}=1\right)$ to $\mathrm{Si}$ $\left(\epsilon_{r}=2.56\right)$, it was observed that the above summations can be truncated for $l_{\phi}=l_{\psi_{0}}=6$. Also, the integrals can be approximated by finite summations of six cells on each side of the central cell $(k$ cell). Due to the orthogonality relationship between the scaling and wavelet functions, for uniform dielectrics (constant $\epsilon_{1}$ throughout the integration domain), these integrals are simplified to $c_{\zeta_{1} \xi_{1}}^{\xi_{1} \xi_{1}, d}\left(l^{\prime}, m^{\prime}\right)=\epsilon_{1} \delta_{l^{\prime}, 0} \delta_{m^{\prime}, 0}$ and $c_{\zeta_{2} \xi_{2}}^{\zeta_{1} \xi_{1}, d}=0$ for $\zeta_{1} \neq \zeta_{2}$ or $\xi_{1} \neq \xi_{2}$, transforming $[\overline{\bar{\epsilon}}]$ to a diagonal matrix. For structures containing dielectric discontinuities, some or all of these integrals have a nonzero value. In this case, the whole geometry has to be preprocessed before the initialization of the time loop, and the coefficients $c_{\zeta_{2} \xi_{2}}^{\zeta_{1} \xi_{1}, d}\left(l^{\prime}, m^{\prime}\right)$ have to be assigned to any cell $(l, m)$ and included in the matrix $[\overline{\bar{\epsilon}}]$. For each cell, the amplitude of these coefficients is compared to the amplitude of the self-term $c_{\phi \phi, d}^{\phi \phi}(0,0)$. If all coefficients are below a threshold (usually $\leq 0.1 \%$ ), they are set to zero and this cell is exempted from the following inversion, otherwise it is included in a new submatrix. For most practical cases, this submatrix has significantly smaller dimensions than $[\overline{\bar{\epsilon}}]$ (usually $\leq 10 \%$ ) and contains only cells close to dielectric discontinuities. The inverse of this matrix is used for the calculation of the $E$ from the $D$ values for each time step. The inversion takes place only once (in the preprocessing stage before the initiation of the simulations), thus it adds only negligible computational overhead to the algorithm. Even for structures with arbitrary dielectric configurations, the number of cells with dense submatrices (density larger than 50\%) is going to be much smaller than the total grid size and the matrix multiplication step for the $E$ calculation will not significantly affect the execution time.

3) Modeling of Excitation: Without loss of generality, the modeling of the excitation for the 2-D algorithm is presented. The two-and-one-half-dimensional (2.5-D) and 3-D algorithm is a direct extension of the 2-D algorithm. 
TABLE II

EXCITATION SCALING DECOMPOSITION COEFFICIENTS

\begin{tabular}{c|c|c|c|c|c|c|c}
\hline$l_{\phi}$ & 0 & 1 & 2 & 3 & 4 & 5 & 6 \\
\hline$c_{\phi}^{e}\left(l_{\phi}\right)$ & 0.91507 & 0.03820 & 0.00963 & -0.00863 & 0.00502 & -0.00268 & 0.00141 \\
\hline
\end{tabular}

In order to apply a point (pulse) excitation $P\left(x_{e}, z_{e}\right)$ for $x_{e}=$ $l_{e} \Delta x, z_{e}=m_{e} \Delta z$, the pulse has to be decomposed in terms of scaling and wavelet functions

$$
\begin{aligned}
& P\left(x_{e}, z_{e}\right) \\
& =\sum_{l_{x, \phi}=-\infty}^{\infty} \sum_{l_{z, \phi}=-\infty}^{\infty} c_{\phi \phi}^{e}\left(l_{x, \phi}, l_{z, \phi}\right) \phi\left(l_{e}+l_{x, \phi}\right) \\
& \quad \cdot \phi\left(m_{e}+l_{z, \phi}\right)+\sum_{l_{x, \phi}=-\infty}^{\infty} \sum_{l_{z, \psi_{o}}=-\infty}^{\infty} c_{\phi \psi_{o}}^{e}\left(l_{x, \phi}, l_{z, \psi_{o}}\right) \\
& \quad \cdot \phi\left(l_{e}+l_{x, \phi}\right) \psi\left(m_{e}+l_{z, \psi_{o}}\right) \\
& \quad+\sum_{l_{x, \psi_{o}}=-\infty}^{\infty} \sum_{l_{z, \phi}=-\infty}^{\infty} c_{\psi_{o} \phi}^{e}\left(l_{x, \psi_{o}}, l_{z, \phi}\right) \psi\left(l_{e}+l_{x, \psi_{o}}\right) \\
& \cdot \phi\left(m_{e}+l_{z, \phi}\right)+\sum_{l_{x, \psi_{o}}=-\infty}^{\infty} \sum_{l_{z, \psi_{o}}=-\infty}^{\infty} c_{\psi_{o} \psi_{o}}^{e}\left(l_{x, \psi_{o}}, l_{z, \psi_{o}}\right) \\
& \cdot \psi\left(l_{e}+l_{x, \psi_{o}}\right) \psi\left(m_{e}+l_{z, \psi_{o}}\right)
\end{aligned}
$$

with

$$
\begin{aligned}
& c_{\phi \phi}^{e_{\phi}}\left(l_{x, \phi}, l_{z, \phi}\right) \\
& =\int_{l_{e}-0.5}^{l_{e}+0.5} \int_{m_{e}-0.5}^{m_{e}+0.5} \phi\left(l_{e}+l_{x, \phi}\right) \phi\left(m_{e}+l_{z, \phi}\right) d z d x \\
& c_{\phi \psi_{o}}^{e}\left(l_{x, \phi}, l_{z, \psi}\right) \\
& \quad=\int_{l_{e}-0.5}^{l_{e}+0.5} \int_{m_{e}-0.5}^{m_{e}+0.5} \phi\left(l_{e}+l_{x, \phi}\right) \psi_{o}\left(m_{e}+l_{z, \psi_{o}}\right) d z d x \\
& c_{\psi_{o} \phi}^{e}\left(l_{x, \psi_{o}}, l_{z, \phi}\right) \\
& \quad=\int_{l_{e}-0.5}^{l_{e}+0.5} \int_{m_{e}-0.5}^{m_{e}+0.5} \psi_{o}\left(l_{e}+l_{x, \psi_{o}}\right) \phi\left(m_{e}+l_{z, \phi}\right) d z d x \\
& c_{\psi_{o} \psi_{o}}^{e}\left(l_{x, \psi_{o}}, l_{z, \psi_{o}}\right) \\
& \quad=\int_{l_{e}-0.5}^{l_{e}+0.5} \int_{m_{e}-0.5}^{m_{e}+0.5} \psi_{o}\left(l_{e}+l_{x, \psi_{o}}\right) \psi_{o}\left(m_{e}+l_{z, \psi_{o}}\right) d z d x .
\end{aligned}
$$

Practically, the summations of (45) can be truncated to a finite number of terms. Usually 6-8 terms on each side of the excitation point per direction can offer an accuracy of representation close to $0.1 \%$. In case the neighboring scaling or wavelet functions are located outside the computational domain (e.g., $l_{e}+l_{x, \phi / \psi_{o}}>n x$ or $l_{e}+l_{x, \phi / \psi_{o}}<0$ for a domain $[0, n x]$ to the $x$-direction), image theory has to be applied for their translation inside the computational grid.

If there is no discontinuity (hard boundary or dielectric interface) in the summation interval of (45), the double integrals of (46) can be split in two single integrals

$$
\begin{aligned}
& c_{\phi \phi}^{e}\left(l_{x, \phi}, l_{z, \phi}\right) \\
& \quad=\int_{l_{e}-0.5}^{l_{e}+0.5} \phi\left(l_{e}+l_{x, \phi}\right) d x \int_{m_{e}-0.5}^{m_{e}+0.5} \phi\left(m_{e}+l_{z, \phi}\right) d z \\
& \quad=c_{\phi}^{e}\left(l_{x, \phi}\right) c_{\phi}^{e}\left(l_{z, \phi}\right)
\end{aligned}
$$

$$
\begin{aligned}
& c_{\phi \psi_{o}}^{e}\left(l_{x, \phi}, l_{z, \psi_{o}}\right) \\
& \quad=\int_{l_{e}-0.5}^{l_{e}+0.5} \phi\left(l_{e}+l_{x, \phi}\right) d x \int_{m_{e}-0.5}^{m_{e}+0.5} \psi_{o}\left(m_{e}+l_{z, \psi_{o}}\right) d z \\
& \quad=c_{\phi}^{e}\left(l_{x, \phi}\right) c_{\psi_{o}}^{e}\left(l_{z, \psi_{o}}\right) \\
& c_{\psi_{o} \phi}^{e}\left(l_{\left.x, \psi_{o}, l_{z, \phi}\right)}\right. \\
& \quad=\int_{l_{e}-0.5}^{l_{e}+0.5} \psi_{o}\left(l_{e}+l_{x, \psi_{o}}\right) d x \int_{m_{e}-0.5}^{m_{e}+0.5} \phi\left(m_{e}+l_{z, \phi}\right) d z \\
& \quad=c_{\psi_{o}}^{e}\left(l_{x, \psi_{o}}\right) c_{\phi}^{e}\left(l_{z, \phi}\right) \\
& c_{\psi_{o} \psi_{o}}^{e}\left(l_{x, \psi_{o}}, l_{z, \psi_{o}}\right) \\
& \quad=\int_{l_{e}-0.5}^{l_{e}+0.5} \psi_{o}\left(l_{e}+l_{x, \psi_{o}}\right) d x \int_{m_{e}-0.5}^{m_{e}+0.5} \psi_{o}\left(m_{e}+l_{z, \psi_{o}}\right) d z \\
& \quad=c_{\psi_{o}}^{e}\left(l_{x, \psi_{o}}\right) c_{\psi_{o}}^{e}\left(l_{z, \psi_{o}}\right),
\end{aligned}
$$

with $c_{\phi}^{e}, c_{\psi_{0}}^{e}$ given in Tables II and III.

Due to the symmetries of the Battle-Lemarie scaling and wavelet functions, the decomposition coefficients have to satisfy the following conditions:

$$
\begin{aligned}
c_{\phi}^{e}\left(l_{\phi}\right) & =c_{\phi}^{e}\left(-l_{\phi}\right), \quad l_{\phi}=-1,-2, \ldots \\
c_{\psi_{o}}^{e}\left(l_{\psi_{o}}\right) & =c_{\psi_{o}}^{e}\left(1-l_{\psi_{o}}\right), \quad l_{\psi_{o}}=0,-1,-2, \ldots
\end{aligned}
$$

If a hard boundary, such as a PEC, is located in the integration domain of the evaluation of the $c^{e}$ coefficients, the image theory should be applied appropriately instead of the above symmetry equations.

For each time step, the excitation scaling and wavelet components have to be superimposed to the respective field values obtained by the MRTD algorithm in order to provide a transparent source similar to FDTD as follows:

$$
\begin{aligned}
& { }_{k} E_{l_{e}+l_{x, \phi}, m_{e}+l_{z, \phi}}^{\phi \phi, \text { total }} \\
& ={ }_{k} E_{l_{e}+l_{x, \phi}, m_{\epsilon}+l_{z, \phi}}^{\phi \phi}+c_{\phi \phi}^{e}\left(l_{x, \phi}, l_{z, \phi}\right) \\
& { }_{k} E_{l_{e}+l_{x, \phi}, m_{e}+l_{z, \psi_{o}}}^{\phi \psi_{o} \text {, total }}
\end{aligned}
$$

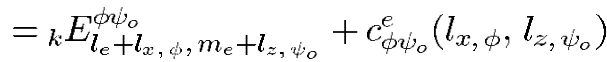

$$
\begin{aligned}
& { }_{k} E_{l_{e}+l_{x, \psi_{o}}, m_{e}+l_{z, \phi}}^{\psi_{o} \phi \text {, total }} \\
& ={ }_{k} E_{l_{e}+l_{x, \psi_{o}}, m_{e}+l_{z, \phi}}^{\psi_{o} \phi}+c_{\psi_{o} \phi}^{e}\left(l_{x, \psi_{o}}, l_{z, \phi}\right) \\
& { }_{k} E_{l_{e}+l_{x, \psi_{o}}, m_{e}+l_{z, \psi_{o}}}^{\psi_{j} \psi_{o}, \text { total }} \\
& ={ }_{k} E_{l_{e}+l_{x, \psi_{o}}, m_{e}+l_{z, \psi_{o}}}^{\psi_{o} \psi_{o}}+c_{\psi_{o} \psi_{o}}^{e}\left(l_{x, \psi_{o}}, l_{z, \psi_{o}}\right) \text {. }
\end{aligned}
$$

For the 2.5-D MRTD algorithm, which requires impulse excitation in time-domain, the above superposition takes place only for the first time step $(t=0)$. Nevertheless, for the 2-D MRTD, it has to be repeated throughout the number of time steps that the excitation is on. The appropriate number of the time steps will depend on the time dependence of the excitation (usually Gaussian, Gabor, or sinusoidal time dependence).

Arbitrary excitation spatial distributions $f(x, z)$ for an area $\left[x_{1}=l_{e, 1} \Delta x, x_{2}=l_{e, 2} \Delta x\right] \times\left[z_{1}=m_{e, 1} \Delta z, z_{2}=m_{e, 2} \Delta z\right]$ can be modeled in a similar way. The spatial distribution has to 
TABLE III

EXCITATION WAVELET DECOMPOSITION COEFFICIENTS

\begin{tabular}{|c|c|c|c|}
\hline i & $a(i)$ & $b_{0}(i)$ & $c_{0}(i)$ \\
\hline $\mathbf{0}$ & 1.29161604157839 & 2.47253977327429 & 0. \\
\hline 1 & -0.155978843323672 & 0.9562282774123074 & $-4.659725793402785 \mathrm{E}-02$ \\
\hline 2 & $5.9606303324687290 \mathrm{E}-02$ & 0.1660591600788887 & 5.453939813583327E-02 \\
\hline 3 & $-2.929157759806890 \mathrm{E}-02$ & $9.392437777679437 \mathrm{E}-02$ & $-3.699957746974982 \mathrm{E}-02$ \\
\hline 4 & $1.5362399457426780 \mathrm{E}-02$ & $3.141444475216036 \mathrm{E}-03$ & $2.057449098775452 \mathrm{E}-02$ \\
\hline 5 & $-8.184462325283712 \mathrm{E}-03$ & $1.349356908709108 \mathrm{E}-02$ & $-1.115303180864957 \mathrm{E}-02$ \\
\hline 6 & $4.3757585552354830 \mathrm{E}-03$ & $-2.858941810094752 \mathrm{E}-03$ & 5.976877725279031E-03 \\
\hline 7 & $-2.342365356649461 E-03$ & $2.778680514115529 \mathrm{E}-03$ & $-3.202621363952005 \mathrm{E}-03$ \\
\hline 8 & 1.252877717042020E-03 & $-1.129446167303586 \mathrm{E}-03$ & $1.714086849566890 \mathrm{E}-03$ \\
\hline 9 & $-6.716635068590737 \mathrm{E}-04$ & $7.071507309377701 \mathrm{E}-04$ & $-9.176508438494196 \mathrm{E}-04$ \\
\hline 10 & $3.583506907489797 \mathrm{E}-04$ & $-3.491267305845643 \mathrm{E}-04$ & $4.911754748072018 \mathrm{E}-04$ \\
\hline 11 & $-1.931321684715780 \mathrm{E}-04$ & $1.952711419194906 \mathrm{E}-04$ & $-2.629253013538502 \mathrm{E}-04$ \\
\hline 12 & 1.019327767057869E-04 & $-1.021304423384722 \mathrm{E}-04$ & $1.407386855875626 \mathrm{E}-04$ \\
\hline 13 & $-5.613943183518454 \mathrm{E}-05$ & $5.531259273864269 \mathrm{E}-05$ & $-7.533840689573666 \mathrm{E}-05$ \\
\hline 14 & $2.834596805928539 \mathrm{E}-05$ & $-2.947330468694831 \mathrm{E}-05$ & $4.033146235099674 \mathrm{E}-05$ \\
\hline 15 & $-1.700348604873522 \mathrm{E}-05$ & $1.572110653438641 \mathrm{E}-05$ & $-2.159462850665844 \mathrm{E}-05$ \\
\hline
\end{tabular}

be sampled with scaling and wavelet functions, giving the new decomposition coefficients

$$
\begin{aligned}
c_{\phi \phi}^{e} & \left(m_{e}, l_{e} ; l_{x, \phi}, l_{z, \phi}\right) \\
= & \int_{l_{e, 1}-0.5}^{l_{e, 2}+0.5} \int_{m_{e, 1}-0.5}^{m_{e, 2}+0.5} f(x, z) \phi\left(l_{e}+l_{x, \phi}\right) \\
& \cdot \phi\left(m_{e}+l_{z, \phi}\right) d z d x \\
c_{\phi \psi_{o}}^{e} & \left(m_{e}, l_{e} ; l_{x, \phi}, l_{z, \psi_{o}}\right) \\
= & \int_{l_{e, 1}-0.5}^{l_{e, 2}+0.5} \int_{m_{e, 1}-0.5}^{m_{e, 2}+0.5} f(x, z) \phi\left(l_{e}+l_{x, \phi}\right) \\
& \cdot{ }_{\left(m_{e}+l_{z, \psi_{o}}\right) d z d x} \\
c_{\psi_{o} \phi}^{e} & \left(m_{e}, l_{e} ; l_{x, \psi_{o}}, l_{z, \phi}\right) \\
= & \int_{l_{e, 1}-0.5}^{l_{e, 2}+0.5} \int_{m_{e, 1}-0.5}^{m_{e, 2}+0.5} f(x, z) \psi_{o}\left(l_{e}+l_{x, \psi_{o}}\right) \\
& \cdot \phi\left(m_{e}+l_{z, \phi}\right) d z d x \\
c_{\psi_{o} \psi_{o}}^{e} & \left(m_{e}, l_{e} ; l_{\left.x, \psi_{o}, l_{z, \psi_{o}}\right)}\right. \\
= & \int_{l_{e, 1}-0.5}^{l_{e, 2}+0.5} \int_{m_{e, 1}-0.5}^{m_{e, 2}+0.5} f(x, z) \psi_{o}\left(l_{e}+l_{x, \psi_{o}}\right) \\
& \cdot \psi_{o}\left(m_{e}+l_{z, \psi_{o}}\right) d z d x,
\end{aligned}
$$

for $l_{e, 1} \leq l_{e} \leq l_{e, 2}$ and $m_{e, 1} \leq m_{e} \leq m_{e, 2}$.

For most simulations, the choice of $-8 \leq l_{x, \phi}, l_{x, \psi_{o}}, l_{z, \phi}$, $l_{z, \psi_{o}} \leq 8$ offers an accuracy close to $0.1 \%$.

Lossy materials can be modeled with MRTD following a procedure similar to the simulation of dielectric interfaces. The additional loss current term $J$ is given by the matrix relationship

$$
[\bar{J}]=[\overline{\bar{\sigma}}][\bar{E}]
$$

where $[\overline{\bar{\sigma}}]$ is the matrix consisting of the sampled conductivity profile for each FDTD cell.

The PML numerical absorber can be easily extended for arbitrary wavelet resolutions in a straightforward way [20]. Due to the entire-domain nature of the Battle-Lemarie expansion basis, a matrix equation has to be solved for the perfectly matched layer (PML) area. For structures with inherent dielectric inhomogeneities, the use of the effective dielectric constant $\epsilon_{\text {eff }}$ provides satisfactory numerical reflection performance.

4) Total Field Reconstruction: Due to the nature of the Battle-Lemarie expansion functions, the total field is a summation of the contributions from the nonlocalized scaling and wavelet functions. For example, the total electric field $E_{x}\left(x_{f}, z_{f}, t_{f}\right)$ with 
$(k-1 / 2) \Delta t<t_{f}<(k+1 / 2) \Delta t,(l-1) \Delta x<x_{f}<l \Delta x$ and $(m-1 / 2) \Delta z<z_{f}<(m+1 / 2) \Delta z$ is calculated in the same way with [4] and [21] by

$$
\begin{aligned}
& E^{x}\left(x_{f}, z_{f}, t_{f}\right) \\
& =\sum_{l^{\prime}=-l_{\phi}}^{l_{\phi}} \phi_{l+l^{\prime}-1 / 2}\left(x_{f}\right) \\
& \cdot\left(\sum_{m^{\prime}=-l_{\phi}}^{l_{\phi}} E_{l+l^{\prime}-1 / 2, m+m^{\prime}}^{x, \phi \phi} \phi_{m+m^{\prime}}\left(z_{f}\right)\right. \\
& \left.+\sum_{m^{\prime}=-l_{\psi_{o}}}^{l_{\psi_{o}}}{ }_{k} E_{l+l^{\prime}-1 / 2 . m+m^{\prime}}^{x, \phi \psi_{o}} \psi_{o, m+m^{\prime}}\left(z_{f}\right)\right) \\
& +\sum_{l^{\prime}=-l_{\psi_{o}}}^{l_{\psi_{o}}} \psi_{o, l+l^{\prime}-1 / 2}\left(x_{f}\right) \\
& \cdot\left(\sum_{m^{\prime}=-l_{\phi}}^{l_{\phi}}{ }_{k} E_{l+l^{\prime}-1 / 2, m+m^{\prime}}^{x, \psi_{o} \phi} \phi_{m+m^{\prime}}\left(z_{f}\right)\right. \\
& \left.+\sum_{m^{\prime}=-l_{\psi_{o}}}^{l_{\psi_{o}}}{ }_{k} E_{l+l^{\prime}-1 / 2 . m+m^{\prime}}^{x, \psi_{o} \psi_{o}} \psi_{o, m+m^{\prime}}\left(z_{f}\right)\right)
\end{aligned}
$$

where $\phi_{l}(x)=\phi((x / \Delta x)-l)$ and $\psi_{l, p}^{r}(x)=2^{r / 2} \psi_{0}$ $\left(2^{r}[(x / \Delta x)-l]-p\right)$ represent the Battle-Lemarie scaling and $r$-resolution wavelet function, respectively, and $r_{\max }$ is the maximum wavelet resolution used in this area of the computational domain. It has been observed that the values $l_{1}=l_{2,0}=l_{3,0}=l_{4,0}=10$ and $l_{2,1}=l_{3,1}=l_{4,1}=6$ offer accuracy close to $0.5 \%$ for most simulations incorporating the first two wavelet resolutions. For the cases of narrow strips with very sharp field discontinuities, the summation limits must increase up to 15-20 terms per direction.

The fact that the MRTD is based on entire-domain basis functions with varying values along each cell offers the unique opportunity of a multipoint field representation per cell. The neighboring scaling and wavelet coefficients can be combined in an appropriate way to calculate the total field value for more than one interior cell points. In this way, MRTD creates a mesh with much larger density than that offered by the nominal number of the cells without increasing the memory requirements. This additional density is very useful in the calculation of the characteristic impedance of planar lines, where even a small field variation can cause a perturbation of the impedance value by 5-10 $\Omega$. On the contrary, FDTD is based on pulse basis functions that have a constant value for each cell, offering a single-point field representation.

5) Generalization for Arbitrary Number of Wavelet Resolutions: For simplicity, wavelets up to $r_{\max }$ resolution are used only to the $z$-direction. The extension to two and three dimensions is straightforward. Since the support of an $\left(r_{o}+1\right)$-resolution wavelet is one-half of the support of an $r_{o}$-resolution wavelet, it is obvious that for each cell, there will exist one zero-resolution wavelet, two one-resolution wavelets, ..., and $2^{r_{\max }-1} r_{\max }$-resolution wavelets. As it was noted earlier, schemes based on a displacement of $H$ and $E$ by $(1 / 2)^{r_{\max }+2}$ instead of $1 / 2$ could provide slightly improved numerical dispersion and stability characteristics. Their derivation is similar to the one presented in this section. After sampling Maxwell's equations with scaling and wavelet functions, expressions similar to (36) are derived. As an example, sampling $\partial E_{x} / \partial t=-(1 / \epsilon) \partial H_{y} / \partial z$ in space and time, the following equations are obtained:

$$
\begin{gathered}
\frac{k+1}{k E_{l+1 / 2, m}^{x, \phi \phi}-{ }_{k} E_{l+1 / 2, m}^{x, \phi \phi}} \\
=-\frac{1}{\epsilon \Delta y}\left(\sum_{m^{\prime}=-n_{a}}^{n_{a}} a\left(m^{\prime}\right)_{k+1 / 2} H_{l+1 / 2, m+m^{\prime}+1 / 2}^{y, \phi \phi}\right. \\
+\sum_{r=0}^{r_{\max }} \sum_{p=0}^{2^{r}-1} \sum_{m^{\prime}=-n_{d_{r}}}^{n_{d_{r}}} d_{r}\left(m^{\prime}, p\right)_{k+1 / 2} \\
\left.\cdot H_{l+1 / 2, m+m^{\prime}+(2 p+1) / 2^{r+1}}^{y, \phi \psi_{r, p}}\right)
\end{gathered}
$$

and

$$
\begin{gathered}
\frac{{ }_{k+1} E_{l+1 / 2, m+\left(2 p^{\prime}+1\right) / 2^{r^{\prime}+1}}^{x, \phi \psi_{r^{\prime}, p^{\prime}}}-{ }_{k} E_{l+1 / 2, m+\left(2 p^{\prime}+1\right) / 2^{r^{\prime}+1}}^{x, \phi \psi_{r^{\prime}, p^{\prime}}}}{\Delta t} c_{m^{\prime}=-n_{c_{r^{\prime}}}\left(m^{\prime}, p^{\prime}\right)_{k+1 / 2} H_{l+1 / 2, m+m^{\prime}+1 / 2}^{y, \phi \phi}}^{n_{c_{r^{\prime}}}}\left(\sum_{r=0}^{r_{\max } 2^{r}-1} \sum_{p=0}^{n_{b_{r^{\prime}}, r}} \sum_{m^{\prime}=-n_{r_{r^{\prime}}, r}} b_{r^{\prime}, r}\left(m^{\prime}, p^{\prime}, p\right)\right. \\
\left.+\sum_{k+1 / 2} H_{l+1 / 2, m+m^{\prime}+(2 p+1) / 2^{r+1}}^{y, \phi \psi_{r, p}}\right)
\end{gathered}
$$

where ${ }_{k} E_{l, m}^{x, \phi \xi}$ and ${ }_{k} H_{l, m}^{y, \phi \xi}$ with $\xi=\phi$ (scaling), $\psi_{r, p}$ (wavelets of $r$ resolution at the $p$-position of the cell) are the coefficients for the electric- and magnetic-field expansions. The indexes $l, m$, and $k$ are the discrete space and time indexes, which are related to the space and time coordinates via $x=l \Delta x, z=m \Delta z$ and $t=k \Delta t$, where $\Delta x, \Delta z$ are the space discretization intervals in the $x$ - and $z$-directions and $\Delta t$ is the time discretization interval. The coefficients $a\left(m^{\prime}\right), c_{r^{\prime}}\left(m^{\prime}, p^{\prime}\right), b_{r^{\prime}, r}\left(m^{\prime}, p^{\prime}, p\right), d_{r}\left(m^{\prime}, p\right)$ are given by (22)-(28). For an accuracy of $0.1 \%$, the parameters $n_{a}, n_{c_{r^{\prime}}}, n_{b_{r^{\prime}, r}}, n_{d_{r}}$ need to take values in the range of 6-12. The summation limits $n_{b_{r^{\prime}, r}}$ get decreasing values as the difference $\left|r^{\prime}-r\right|$ gets larger due to the different scales of the wavelet domains reaching the minimum value of one even for $\left|r-r^{\prime}\right|=2$.

To model a dielectric discontinuity, the starting point is the constitutive relationship $D=\epsilon E$ that derives the following equations for $D_{x}$ :

$$
\begin{aligned}
& { }_{k} D_{l, m}^{x, \phi \phi} \\
& =\sum_{l^{\prime}=-l_{\phi}}^{l_{\phi}}\left(\sum_{m^{\prime}=-l_{\phi}}^{l_{\phi}} c_{\phi \phi}^{\phi \phi, d}\left(l^{\prime}, m^{\prime}\right)_{k} E_{l+l^{\prime}, m+m^{\prime}}^{x, \phi \phi}\right. \\
& \left.+\sum_{r=0}^{r_{\max }} \sum_{p=0}^{2^{r}-1} \sum_{m^{\prime}=-l_{\psi_{r}, p}}^{l_{\psi_{r, p}}} c_{\phi \psi_{r, p}}^{\phi \phi, d}\left(l^{\prime}, m^{\prime}\right)_{k} E_{l+l^{\prime}, m+m^{\prime}}^{x, \phi \psi_{r, p}}\right)
\end{aligned}
$$


and

$$
\begin{aligned}
{ }_{k} D_{l, m}^{x, \phi \psi_{r^{\prime}, p^{\prime}}} & \\
=\sum_{l^{\prime}=-l_{\phi}}^{l_{\phi}} & \left(\sum_{m^{\prime}=-\xi_{\phi}}^{\xi_{\phi}} c_{\phi \phi}^{\phi \psi_{r^{\prime}, p^{\prime}}, d}\left(l^{\prime}, m^{\prime}\right)_{k} E_{l+l^{\prime}, m+m^{\prime}}^{x, \phi \phi}\right. \\
& \left.+\sum_{r=0}^{r_{\max }} \sum_{p=0}^{2^{r}-1} \sum_{m^{\prime}=-\xi_{\psi_{r}, p}}^{\xi_{\psi_{r}, p}} c_{\phi \psi_{r, p}}^{\phi \psi_{r^{\prime}, p^{\prime}}, d}\left(l^{\prime}, m^{\prime}\right)_{k} E_{l+l^{\prime}, m+m^{\prime}}^{\psi_{r, p}}\right)
\end{aligned}
$$

where the $c_{\xi_{1} \xi_{2}}^{\zeta_{1} \zeta_{2}}, d$ coefficients are defined by (46). For each time step, $D$ is calculated by $H$ through the discretized Maxwell's equations and $E$ is derived by the solution of $[D]=[\overline{\bar{\epsilon}}][E]$.

In order to implement a pulse excitation $P\left(x_{e}, z_{e}\right)$ at $x_{e}=$ $l_{e} \Delta x$ and $z_{e}=m_{e} \Delta z$, the space pulse is expressed in terms of scaling and wavelet functions

$$
\begin{aligned}
& P\left(x_{e}, z_{e}\right) \approx \sum_{l_{x, \phi}=-\infty}^{\infty} \phi\left(l_{e}+l_{x, \phi}\right) \\
&\left(\sum_{l_{z, \phi}=-\infty}^{\infty} c_{\phi \phi}^{e}\left(l_{x, \phi}, l_{z, \phi}\right) \phi\left(m_{e}+l_{z, \phi}\right)\right. \\
&+\sum_{r=0}^{r_{\max }} \sum_{p=0}^{2^{r}-1} \sum_{l_{z, \psi_{r, p}}=-\infty}^{\infty} c_{\phi \psi_{r, p}}^{e}\left(l_{x, \phi}, l_{z, \psi_{r, p}}\right) \\
&\left.\cdot \psi_{r, p}\left(m_{e}+l_{z, \psi_{r, p}}\right)\right)
\end{aligned}
$$

where the coefficients $c_{\phi \phi}^{e}, c_{\phi \psi_{r, p}}^{e}$ can be calculated similarly as shown in Section III-B.3.

The total electric field $E^{x}\left(x_{f}, z_{f}, t_{f}\right)$ with $(l-1 / 2) \Delta x<$ $x_{f}<(l+1 / 2) \Delta x,(m-1 / 2) \Delta z<z_{f}<(m+1 / 2) \Delta z$, and $(k-1 / 2) \Delta t<t_{f}<(k+1 / 2) \Delta t$ is calculated summing up the effect of all contributing scaling and wavelet coefficients. Thus, in a way parallel to Section III-B.4

$$
\begin{aligned}
& E^{x}\left(x_{f}, z_{f}, t_{f}\right) \\
& =\sum_{l^{\prime}=-l_{\phi}}^{l_{\phi}} \phi_{l+l^{\prime}-1 / 2}\left(x_{f}\right) \\
& \quad\left(\sum_{m^{\prime}=-l_{\phi}}^{l_{\phi}} E_{l+l^{\prime}+1 / 2, m+m^{\prime}}^{x, \phi \phi} \phi_{m+m^{\prime}}\left(z_{f}\right)\right. \\
& \quad+\sum_{r=0}^{r_{\max }} \sum_{p=0}^{2^{r}-1} \sum_{m^{\prime}=-l_{\psi_{r}, p}}^{l_{\psi_{r, p}}} E_{l+l^{\prime}+1 / 2, m+m^{\prime}+(2 p+1) / 2^{r+1}}^{x, \phi \psi_{r, p}} \\
& \left.\cdot \psi_{m+m^{\prime}+(2 p+1) / 2^{r+1}}^{r, p}\left(z_{f}\right)\right)
\end{aligned}
$$

where $\phi_{m}(x)=\phi((x / \Delta x)-m)$ and $\psi_{m}^{r, p}(x)=\psi_{m}^{r}((x / \Delta x)-$ $p)=2^{r / 2} \psi_{m}^{0}\left(2^{r}(x / \Delta x)-p\right)$ represent the Battle-Lemarie scaling and $r$-resolution wavelet function, respectively.

6) Time-Adaptive Gridding: The MRA is based on the fact that the wavelets increase the local resolution of the expansion. Each added wavelet resolution virtually is equivalent to the use of a denser grid with one-half cell size. In addition, wavelets have significant values close to discontinuities or near

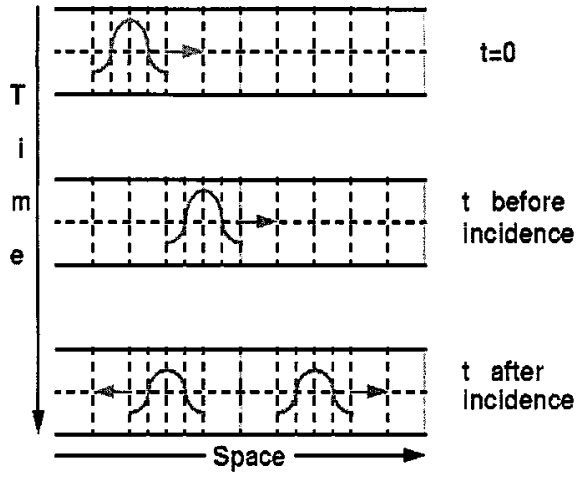

Fig. 9. Time- and space-adaptive grid.

regions of fast field variation since they contain only high-frequency spatial components. There are many different ways to take advantage of these wavelet characteristics in order to create a space and time adaptive gridding algorithm. In digital signal processing, thresholding of the wavelet coefficients over a specific time and space window (5-10 points) contribute significant memory economy (a factor between 4-8 in comparison to scaling-only expansions), but increase the implementation complexity and execution time. Sometimes the added computational overhead is greater than the previous execution time.

The simplest way to create a dynamically changing grid is to threshold the wavelet components to a fraction (usually $\leq 0.1 \%$ ) of the scaling function at the same cell (space adaptiveness) and/or to an absolute threshold (usually 0.0001 or a number smaller than $1 / 10000$ of the peak of the excitation time-domain function) [5], [17]. This comparison is repeated for each time step (time adaptiveness). All components below this threshold are eliminated from the subsequent calculations. This is the simplest thresholding algorithm. It does not add any significant overhead in execution time (usually $\leq 10 \%$ ), but it offers only a moderate (pessimistic) economy in memory (a factor close to two). Also, this algorithm allows for the dynamic memory allocation in its programming implementation by using the appropriate programming languages (e.g., C).

The principles of the dynamically changing time- and spaceadaptive grid are demonstrated in Fig. 9. A pulse is propagating from the left-hand side to the right-hand side in a partially filled parallel-plate waveguide. For $t=0$, the wavelets are localized at the excitation area. They follow the propagating pulse ( $t$ before the incidence to the dielectric interface), creating a moving dense subgrid. After the pulse has been split in reflected and transmitted pulses, the wavelets increase the grid resolution only around these pulses. Elsewhere the wavelet components have negligible values and are ignored.

\section{APPLICATIONS OF MRTD}

As it was stressed above, the most important feature of MRTD is the capability of adaptive gridding, which allows for the modeling of very fine geometrical details using cells close to Nyquist limit everywhere else. Since there are more than one points per MRTD cell, it is possible calculating multiple wavelet resolutions to make use of cell sizes larger than the Nyquist limit, though the effective cell size (real cell size/subpoints per cell) 


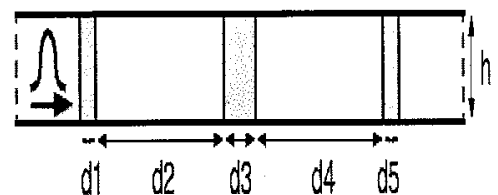

Fig. 10. Parallel-plate five-stage filter.

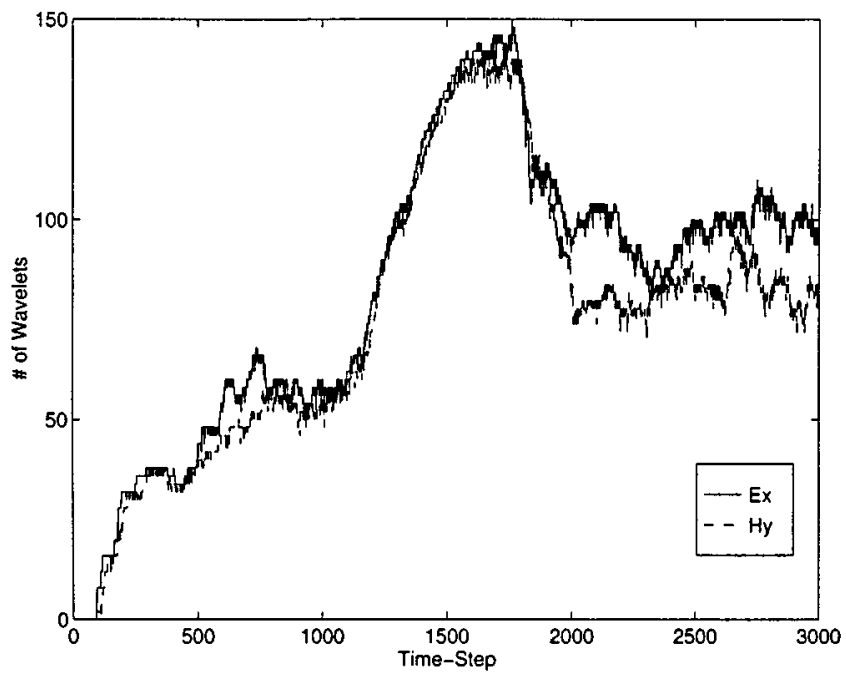

Fig. 11. Nonzero wavelets' number.

is always smaller than this limit. For simplicity, one resolution of wavelets was used, though more wavelet resolutions can be added in a straightforward way.

Initially, the adaptive MRTD gridding with arbitrary resolutions of wavelets was applied to the simulation of a five-stage parallel-plate filter of Fig. 10 [17]. A Gabor function $0-4 \mathrm{GHz}$ propagated from the left-hand side to the right-hand side. The input and output stages had $\epsilon_{r}=12.5$ and the intermediate stages have $\epsilon_{r}=50.5$ (stages with $d_{1}=d_{5}=0.5 \mathrm{~mm}$ and $d_{3}=2 \mathrm{~mm}$ ) and $\epsilon_{r}=1$. (Stages with $d_{2}=d_{4}=14 . \mathrm{mm}$.) The capability of MRTD to model more than one dielectrics per cell, as was described in Section III-B-2, allowed for the use of a very large cell size for the scaling functions, though the wavelets accurately described the intracell dielectric interfaces. The total length to the vertical direction was $4.8 \mathrm{~mm}$. To study the effect of the numerical dispersion, the simulated longitudinal direction was stretched to $600 \mathrm{~mm}$. PML regions of 16 cells with $R_{\max }=10^{-4}$ terminated the grid and wavelets of zero resolution were used to the longitudinal direction. The structure was analyzed by using an FDTD grid of $8 \times 1600$ cells, a scaling-only MRTD grid of $2 \times 400$ cells, and an adaptive (scaling + wavelets) MRTD grid of $2 \times 200$ cells. The relative threshold had the value of $0.01 \%$ and the absolute threshold was equal to $10^{-4}$. The maximum number of wavelets required during the 3000 time steps of the simulation was 153 (Fig. 11), offering an economy by $30 \%$ in comparison to the scaling only grid and by a factor of 23 in comparison to the FDTD scheme. The accuracy in the calculation of the $S$-parameters was similar for all three schemes, as can be observed from Fig. 12. Again, the time- and space-adaptive character of the proposed gridding was demonstrated in Fig. 13 with the $E_{x}$-field space distribution for $t=1000$ time steps. The wavelets follow the reflected
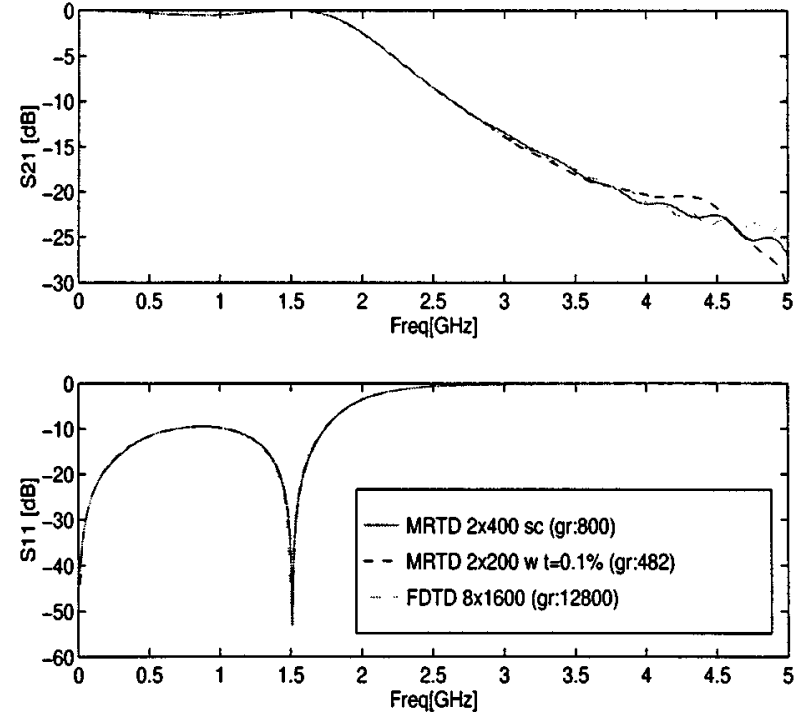

Fig. 12. $S$-parameters of the filter.
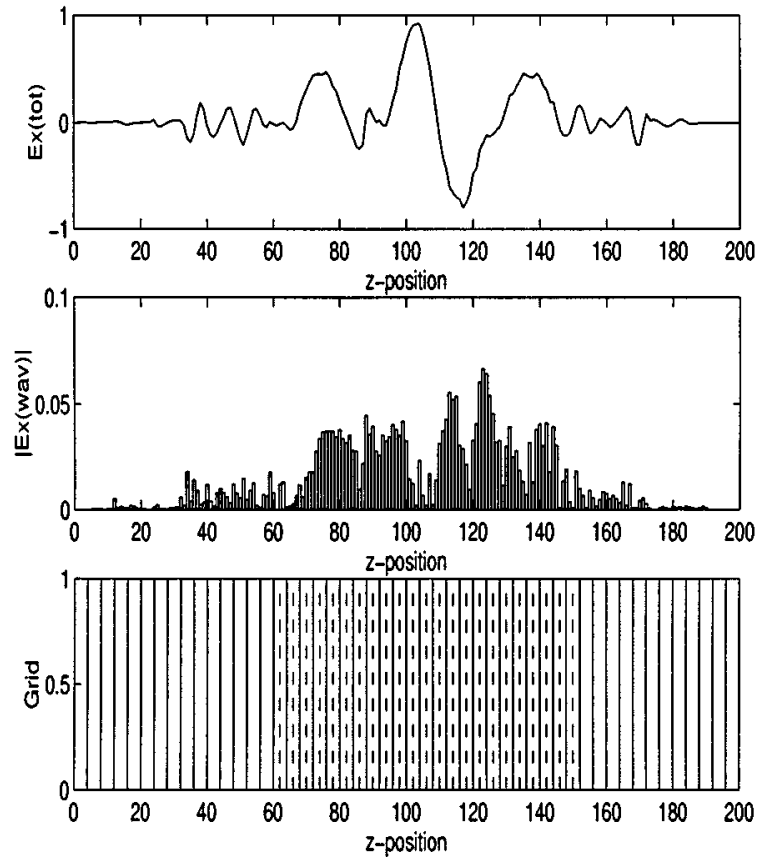

Fig. 13. Adaptive grid demonstration $(t=1000$ steps $)$.

and transmitted pulses after the incidence to the dielectric interfaces and have negligible values elsewhere. The location and number of wavelet coefficients with significant values are different for each time step; something that creates a dense mesh in regions of strong field variations, while maintaining a much coarser mesh for the other cells.

It could be claimed that the variable FDTD grid might be a simpler and more versatile alternative for the full-wave simulation of complex RF structures. Nevertheless, it is a static grid in the time domain. On the contrary, the MRTD grid is updated for each time step based on the status of the electromagnetic-field propagation, something that leads to the minimization of the required computational effort. In addition, the Battle-Lemarie-based MRTD scheme exhibits dispersion 


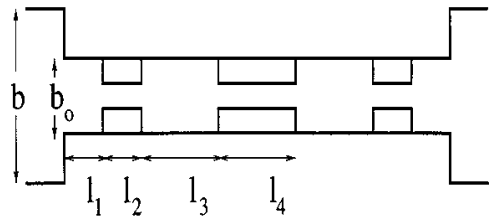

Fig. 14. Optimized evanescent-mode filter geometry.

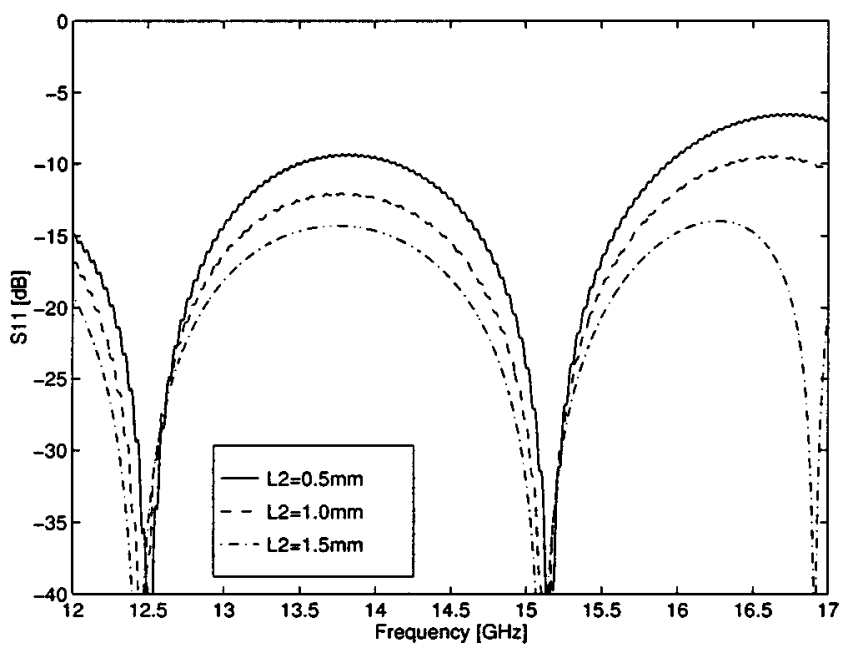

Fig. 15. Parametric variation of $S_{21}$ for $l_{2}$.

characteristics of a 22nd-order FDTD scheme, though the variable-grid-based FDTD still is a second-order discrete algorithm.

After validating the performance of the adaptive gridding, MRTD was extended and used for the optimization of the 3-D evanescent-mode $E$-plane finned waveguide bandpass filter shown in Fig. 14. A WR90 waveguide $(22.86 \mathrm{~mm} \times 10.16 \mathrm{~mm})$ was used at the input and output stages and a rectangular waveguide with a cross section of $7.06 \mathrm{~mm} \times 6.98 \mathrm{~mm}$ was used as the housing of the filter. The width of the fins was chosen to be equal to the waveguide width $a=w=7.06 \mathrm{~mm}$ (dimensions to the direction that is vertical to the plane of Fig. 14), and the initial values of the optimization longitudinal geometrical parameters were $l_{1}=l_{2}=0.5 \mathrm{~mm}, l_{3}=7.75 \mathrm{~mm}$, and $l_{4}=0.94 \mathrm{~mm}$. An MRTD adaptive $20 \times 20 \times 389$ grid and 85000 steps were simulated. A relative threshold of $0.5 \%$ was employed. This structure would have required a huge grid using the conventional FDTD algorithm due to the geometrical details. Using MRTD led to memory economies by 3-6 per dimension and execution time savings by a factor of 2.5 and allowed for the derivation of parametric design curves similar to Fig. 15.

\section{CONCLUSION}

A dynamically changing space- and time-adaptive meshing algorithm based on a MRTD scheme in two dimensions and on absolute and relative thresholding of the wavelet values has been proposed and has been applied to the numerical analysis of various nonhomogeneous waveguide geometries. A mathematically correct way of modeling boundary conditions, launching the excitation, and reconstructing the field, as well as guidelines for the stability and dispersion performance, have been presented and evaluated for expansion using entire-domain scaling and wavelet functions (cubic-spline Battle-Lemarie). In comparison to Yee's conventional FDTD scheme, the proposed scheme offers memory savings by a factor of 3-6 per dimension and significantly smaller execution time maintaining a similar accuracy. The above algorithm can be effectively extended to 2.5-D and 3-D complex microwave problems.

\section{REFERENCES}

[1] K. S. Yee, "Numerical solution of initial boundary value problems involving Maxwell's equations in isotropic media," IEEE Trans. Antennas Propagat., vol. AP-14, pp. 302-307, 1966.

[2] A. Taflove, Computational Electrodynamics. Norwood, MA: Artech House, 1995.

[3] M. Krumpholz and P. Russer, "Two-dimensional FDTD and TLM," Int. J. Numer. Modeling, vol. 7, no. 2, pp. 141-153, Feb. 1993.

[4] M. Krumpholz and L. P. B. Katehi, "MRTD: New time domain schemes based on multiresolution analysis," IEEE Trans. Microwave Theory Tech., vol. 44, pp. 555-561, Apr. 1996.

[5] E. Tentzeris, J. Harvey, and L. P. B. Katehi, "Time adaptive time-domain techniques for the design of microwave circuits," IEEE Microwave Guided Wave Lett., vol. 9, pp. 96-98, Mar. 1999.

[6] L. Roselli, E. Tentzeris, and L. P. B. Katehi, "Nonlinear circuit characterization using a multiresolution time-domain technique (MRTD)," in Proc. IEEE MTT-S Int. Microwave Symp. Dig., 1998, pp. 1387-1390.

[7] K. Goverdhanam, E. Tentzeris, M. Krumpholz, and L. P. B. Katehi, "An FDTD multigrid based on multiresolution analysis," in Proc. IEEE AP-S Symp. Dig., 1996, pp. 352-355.

[8] M. Fujii and W. J. R. Hoefer, "Formulation of a Haar-wavelet based multiresolution analysis similar to the 3-D FDTD method," in Proc. IEEE MTT-S Int. Microwave Symp. Dig., 1998, pp. 1393-1396.

[9] C. Sarris and L. P. B. Katehi, "Multiresolution Time Domain (MRTD) schemes with space-time Haar wavelets," in Proc. IEEE MTT-S Int. Microwave Symp. Dig., 1999, pp. 1459-1462.

[10] S. Mallat, "Multiresolution representation and wavelets," Ph.D. dissertation, Dept. Math., Univ. Pennsylvania, Philadelphia, PA, 1988.

[11] Y. Meyer, Ondelettes et Fonctions Splines. Paris, France: Ecole Polytech., 1986.

[12] I. Daubechies, "Ten lectures on wavelets," in Society for Industrial and Applied Mathematics. Philadelphia, PA: SIAM, 1992.

[13] G. Battle, "A block spin construction of ondelettes-Part I: Lemarie functions," Commun. Math. Phys., vol. 110, pp. 601-615, 1987.

[14] P. G. Lemarie, "Ondelettes a localization exponentielles," J. Math. Pures Appl., vol. 67, pp. 227-236, 1998.

[15] A. Haar, "Zur Theorie der Orthogonalen Funktionsysteme," Math. Ann., vol. 69, pp. 331-371, 1910 .

[16] D. Pozar, Microwave Engineering. Reading, MA: Addison-Wesley, 1991, ch. 4

[17] E. Tentzeris, R. Robertson, A. Cangellaris, and L. P. B. Katehi, "Spaceand time-adaptive gridding using MRTD," in Proc. IEEE MTT-S Int. Microwave Symp. Dig., 1997, pp. 337-340.

[18] E. Tentzeris, R. Robertson, J. Harvey, and L. P. B. Katehi, "Stability and dispersion analysis of Battle-Lemarie based MRTD schemes," IEEE Trans. Microwave Theory Tech., vol. 47, pp. 1004-1013, July 1999.

[19] R. L. Robertson, E. Tentzeris, and L. P. B. Katehi, "Modelling of dielectric-loaded cavities using MRTD," Int. J. Numer. Modeling (Special Issue), vol. 11, pp. 55-68, 1998.

[20] E. Tentzeris, R. Robertson, J. Harvey, and L. P. B. Katehi, "PML absorbing boundary conditions for the characterization of open microwave circuit components using MRTD," IEEE Trans. Antennas Propagat., vol. 47, pp. 1709-1715, Nov. 1999.

[21] E. Tentzeris, R. L. Robertson, M. Krumpholz, and L. P. B. Katehi, “Application of MRTD to printed transmission lines," in Proc. IEEE MTT-S Int. Microwave Symp. Dig., 1996, pp. 573-576. 


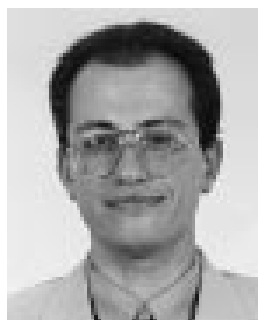

Emmanouil M. Tentzeris (M'90) received the Diploma degree in electrical engineering and computer science (suma cum laude) from the National Technical University, Athens, Greece, in 1992, and the M.S. and Ph.D. degrees in electrical engineering and computer science from The University of Michigan at Ann Arbor, in 1993 and 1998.

In 1998, he joined the School of Electrical and Computer Engineering, Georgia Institute of Technology, Atlanta, as an Assistant Professor, and is currently the leader of the Novel Integration Techniques Subthrust of the Broadband Hardware Access Thrust of the Yamacraw Initiative of the State of Georgia and the Packaging Research Center Thrust Leader for RF/Wireless Packaging. He has established academic programs in adaptive numerical electromagnetics, RF and wireless applications, and RF microelectromechanical (MEMS) modeling. He has authored or co-authored over 70 publications, three book chapters, and numerous invited papers

Dr. Tentzeris was the 1999 Technical Program co-chair of the 54th ARFTG Conference and is currently a member of the Technical Program Committees of the IEEE Microwave Theory and Techniques (IEEE MTT-S) International Microwave Symposium (IMS) and the IEEE Antennas and Propagation Society (IEEE AP-S) Symposium. He is the vice-chair of the IEEE CPMT TC16 (RF Subcommittee). He was the recipient of the 2000 National Science Foundation CAREER Award, the 1997 International Microelectronics and Packaging Society (IMAPS) Best Paper Award, and the 2001 Applied Computational Electromagnetics (ACES) Best Student Paper Award.

Andreas Cangellaris (M'86-SM'97-F'00) received the Diploma degree in electrical engineering from the University of Thesalloniki, Thesalloniki, Greece, in 1981, and the M.S. and Ph.D. degrees in electrical engineering from the University of California at Berkeley, in 1983 and 1985, respectively.

$\mathrm{He}$ is currently a Professor of electrical and computer engineering at the University of Illinois at Urbana-Champaign. Prior to joining the University of Illinois at Urbana-Champaign in 1997, he was with the faculty of the Department of Electrical and Computer Engineering, University of Arizona, from 1987 to 1997. After finishing his doctoral studies, and from 1985 to 1987, he was a Senior Research Engineer in the Department of Electronics Engineering, General Motors Research Laboratories, Warren, MI. His research interests and contributions are in two major areas. The first is the area of applied computational electromagnetics. The second is the area of modeling and simulation for high-speed interconnection and package electrical analysis. He has authored and co-authored over 100 scientific journal and conference papers in these areas.

Prof. Cangellaris is co-founder of the IEEE Topical Meeting on Electrical Performance of Electronic Packaging, which is sponsored jointly by the IEEE Microwave Theory and Techniques Society (IEEE MTT-S) and the IEEE Components, Packaging and Manufacturing Technology Society. He served as the meeting co-chair for the first three years. Since then, he serves as a member of its Technical Program Committee. Today, this meeting is considered the premier conference on package electrical analysis and design. He is also an active participant in the Electronic Components and Technology Conference (ECTC), as a member of the Technical Program Subcommittee on Modeling and Simulation. In addition, he serves as member of the Technical Program Committee for the IEEE MTT-S International Microwave Symposium. In 1998, he was the general chairman for the 8th Biennial Conference on Electromagnetic Field Computation, Tucson, AZ, which is sponsored by the IEEE Magnetics Society.

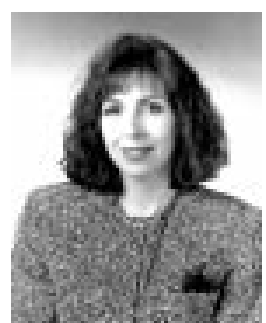

Linda P. B. Katehi (S'81-M'84-SM'89-F'95) received the B.S.E.E. degree from the National Technical University of Athens, Athens, Greece, in 1977, and the M.S.E.E. and Ph.D. degrees from the University of California at Los Angeles, in 1981 and 1984, respectively.

In September 1984, she joined the faculty of the Electrical Engineering and Computer Science Department, The University of Michigan at Ann Arbor, as an Assistant Professor, and then became an Associate Professor in 1989 and Professor in 1994. She has served in many administrative positions, including Director of Graduate Programs, College of Engineering (1995-1996), Elected Member of the College Executive Committee (1996-1998), Associate Dean For Graduate Education (1998-1999), and Associate Dean for Academic Affairs (1999-2001). Since January 2002, she has been the Dean of the Schools of Engineering, Purdue University, West Lafayette, IN. She has authored or co-authored 410 papers published in refereed journals and symposia proceedings and holds four U.S. patents. She has also generated 20 Ph.D. students.

Dr. Katehi is a member of the IEEE Antennas and Propagation Society (IEEE AP-S), the IEEE Microwave Theory and Techniques Society (IEEE MTT-S), Sigma Xi, Hybrid Microelectronics, and URSI Commission D. She was a member of the IEEE AP-S AdCom (1992-1995). She was an associate editor for the IEEE TRANSACTIONS ON MICROWAVE THEORY AND TECHNIQUES and the IEEE TRANSACTIONS ON ANTENNAS AND PROPAGation. She was the recipient of the 1984 IEEE AP-S W. P. King (Best Paper Award for a Young Engineer), the 1985 IEEE AP-S S. A. Schelkunoff Award (Best Paper Award), the 1987 National Science Foundation Presidential Young Investigator Award, the 1987 URSI Booker Award, the 1994 Humboldt Research Award, the 1994 University of Michigan Faculty Recognition Award, the 1996 IEEE MTT-S Microwave Prize, the 1997 International Microelectronics and Packaging Society (IMAPS) Best Paper Award, and the 2000 IEEE Third Millennium Medal.

James Harvey (M'91-SM'99) is currently a Research Program Manager at the Army Research Office, Research Triangle Park, NC, where his primary responsibility is within the fields of electromagnetics, antennas and antenna structures, innovative microwave and millimeter-wave circuit integration, low-power/minimum-power system design, and landmine detection. His programs include a focus on small multifrequency multifunctional antennas for U.S. Army vehicles, radio propagation over complex terrain affecting data communications, and new millimeter-wave circuit integration techniques such as spatial power combining, micromachining, and advanced electromagnetic (EM) calculational techniques. His personal research interests are in the fields of quasi-optics and multiresolution analysis of EM structures. 